\title{
Observer-Based Decentralized Tracking Control with Preview Action for a Class of Nonlinear Interconnected Systems
}

\begin{abstract}
Xiao Yu
School of Science, Shandong Jianzhu University, Jinan 250101, China

Correspondence should be addressed to Xiao Yu; yuxiao201319@163.com

Received 17 August 2019; Accepted 4 December 2019; Published 29 January 2020

Guest Editor: Jorge-Antonio Lopez-Renteria

Copyright (C) 2020 Xiao Yu. This is an open access article distributed under the Creative Commons Attribution License, which permits unrestricted use, distribution, and reproduction in any medium, provided the original work is properly cited.

In this paper, for the first time, the observer-based decentralized output tracking control problem with preview action for a class of interconnected nonlinear systems is converted into a regulation problem for $N$ augmented error subsystems composed of the tracking error dynamics, the difference equation of the state observer, and the available future reference trajectory dynamics associated with each individual subsystem. The developed innovative formulation of an observer-based decentralized preview tracking control scheme consists of the integral control action, the observer-based state feedback control action, and the preview action of the desired trajectory. The controller design feasibility conditions are formulated in terms of a linear matrix inequality (LMI) by using the Lyapunov function approach to ensure the existence of the suggested observer-based decentralized control strategy. Furthermore, both decentralized observer gain matrices and decentralized tracking controller gain matrices can be efficiently and simultaneously computed through a one-step LMI procedure. Stability analysis of the closed-loop augmented subsystem is carried out to illustrate that all tracking errors asymptotically converge toward zero. Finally, a numerical example is provided to demonstrate the effectiveness of the suggested control approach.
\end{abstract}

\section{Introduction}

In the past few years, the output tracking control for interconnected systems that are composed of interconnected lower-dimensional individual subsystems has attracted a great deal of interests from researchers. At present, such systems appear in many practical applications such as telecommunication and transportation networks [1], power systems [2], wind turbines [3], complex mobile robots [4], 3DOF helicopters [5], and so on. It is well known that compared to the traditional centralized control strategy, the decentralized scheme is considered to be the opposite design method, resulting in satisfactory output tracking with a straightforward and more economical implementation $[6,7]$. As a matter of fact, the main feature of decentralized control lies in designing the control law for each individual subsystem utilizing only locally available information. The collection of these control laws constitutes the control structure of the whole interconnected system. Some interesting results in this field are presented in $[5,8-14]$.
It should be pointed out that, first, the previously mentioned decentralized control scheme involves the state feedback. In most practical problems, however, the state variable knowledge of each subsystem is not always fully available, mainly due to the technical and/or economic limitations. To deal with this issue, a feasible alternative is to reconstruct the inaccessible state variables via a dynamic state observer. Much effort has been devoted to this area, and we refer the readers to [15-18]. Among them, several studies concentrate on the state reconstruction problem of interconnected systems. The interconnected state observer, where the interconnection information between local observers is used to design the observer, is studied in $[18,19]$. However, the state reconstruction in a decentralized framework, which depends only on the local control input and output of the subsystem, provides an efficient and low-cost observer design to replace the usual state feedback for interconnected systems $[10,11]$.

Second, when the future information of the desired trajectory is known beforehand, i.e., it is previewable, the 
tracking performance of the closed-loop system can be effectively improved by a preview control technique [2022]. The salient feature of the preview control design is that it inserts the preview compensation action that incorporates the available future knowledge of the desired trajectory into the implemented control structure. Up until now, there have been systematic research frameworks on the preview controller design for the linear, the uncertain, and the stochastic systems. We refer the readers to [21-25] for some recent work in this area. In addition to the theoretical developments, many experimental studies have demonstrated that the preview control has potential applications in the fields of active suspension systems [26, 27], intelligent electric vehicles [28, 29], robots $[30,31]$, water tank level control systems [32], brushless DC motors [33], etc.

In this paper, the observer-based decentralized tracking controller design with preview action for interconnected nonlinear systems is studied for the first time. It should be noted that observer-based controller design is not an easy task in this situation as the popular separation principle may not be applicable $[11,34]$. To address this problem, the decentralized tracking control and decentralized observer design are simultaneously taken into account to ensure that the output of each subsystem asymptotically tracks the desired trajectory. For this purpose, we combine the state observation equation, the tracking error dynamics, and the known future desired trajectory dynamics for every individual subsystem to derive a new augmented error subsystem. The integral control action is naturally included using the classical difference approach and the tracking error dynamics. Furthermore, the global augmented error dynamics of the overall interconnected systems can be obtained using the lifting method, which converts the decentralized tracking control problem into a decentralized stabilization problem. With some special mathematical derivations, the proposed controller design method is formulated as an LMI feasibility problem, which can be efficiently resolved through a one-step procedure for the simultaneous computation of the decentralized tracking control gain matrices and the decentralized observation gain matrices. Finally, two numerical examples are provided to verify the theoretical results. The main contributions of the present study are concluded from the following two folds. First, a novel observer-based decentralized tracking control scheme with preview action is proposed for a class of Lipschitz nonlinear interconnected systems that are widely employed in practical applications. Second, the tracking control synthesis procedure is simplified by solving the observer gain and the tracking controller gain simultaneously with only one LMI problem, which effectively reduces the design complexity and the computational load.

The rest of the paper is organized as follows. Section 2 gives the problem formulation and control objective. In Section 3, the construction of the augmented error system is developed. In Section 4, the observer-based decentralized preview tracking controller design method is proposed for a class of nonlinear interconnected systems. Simulation results are provided in Section 5. Section 6 concludes this paper.

Notations. Throughout this paper, $R^{n}$ denotes the $n$-dimensional Euclidean space, and $R^{n \times m}$ denotes the $n \times m$ matrix space. The notation $P>0$ (or $P<0$ ) is used to define a symmetric positive-definite (or negative-definite) matrix. For matrices $P$ and $Q, P>Q$ stands for $P-Q>0$. The notation $\operatorname{diag}(A, B)$ represents a block-diagonal matrix whose diagonal elements are matrices $A$ and $B . M^{T}$ denotes the transpose of matrix $M$. $\operatorname{sym}(A)$ is used to represent $A+A^{T}$. $\|\cdot\|$ denotes the Euclidean norm. In large matrix expressions, the symbol “*” replaces terms that are induced by symmetry.

\section{Problem Formulation}

Consider the following interconnected system described by the connection of $N$ subsystems as follows:

$$
\left\{\begin{array}{l}
x_{i}(k+1)=A_{i} x_{i}(k)+B_{i} u_{i}(k)+f_{i}(x(k)), \\
y_{i}(k)=C_{i} x_{i}(k), \\
i=1,2, \ldots, N
\end{array}\right.
$$

where $A_{i} \in R^{n_{i} \times n_{i}}, \quad B_{i} \in R^{n_{i} \times m_{i}}, \quad$ and $C_{i} \in R^{p_{i} \times n_{i}}$ denote the state matrix, the control matrix, and the output matrix of each subsystem, respectively; $x_{i}(k)=$ $\left[x_{i 1}(k) x_{i 2}(k) \cdots x_{i n_{i}}(k)\right]^{T} \in R^{n_{i}}$ is the state vector of the $i$ th subsystem; $u_{i}(k) \in R^{m_{i}}$ is the control input of the $i$ th subsystem; $y_{i}(k) \in R^{p_{i}}$ is the output vector of the $i$ th subsystem; $\quad x(k)=\left[\begin{array}{lllll}x_{1}^{T}(k) & x_{2}^{T}(k) & \cdots & x_{N}^{T}(k)\end{array}\right]^{T} \in R^{n}$ is the state vector of the overall interconnected system; and $f_{i}(x(k)) \in R^{n_{i}}$ stands for the vector of nonlinear interconnection terms of the $i$ th subsystem and other subsystems.

Assumption 1. The nonlinear interconnection term $f_{i}(x)$ $(i=1,2, \ldots, N)$ is globally Lipschitz with respect to $x$, i.e., there exists a constant $\gamma_{i}>0$ such that for all $x, \bar{x} \in R^{n}$ the following holds:

$$
\left\|f_{i}(x)-f_{i}(\bar{x})\right\| \leq \gamma_{i}\|x-\bar{x}\|
$$

Remark 1. Assumption 1 is referred to as a globally Lipschitz condition. The structure of the nonlinearity satisfying this assumption extensively represents the nonlinear interconnection terms of a broad class of industrial processes; among them, a typical example is the multimachine power system $[2,7]$, and it has been widely used in the design of the decentralized control for complex interconnected systems $[7,8,10,11]$. In particular, if an interconnected term $f_{i}(x)$ is a linear function denoted by $f_{i}(x)=H_{i} x$ and $H_{i} \in R^{n_{i} \times n}$, the restriction (2) is naturally satisfied with $\gamma_{i}=\left\|H_{i}\right\|$.

The global system is characterized by the following state representation:

$$
\left\{\begin{array}{l}
x(k+1)=A x(k)+B u(k)+f(x(k)), \\
y(k)=C x(k)
\end{array}\right.
$$


where

$$
\begin{aligned}
x(k) & =\left[\begin{array}{c}
x_{1}(k) \\
x_{2}(k) \\
\vdots \\
x_{N}(k)
\end{array}\right], \\
u(k) & =\left[\begin{array}{c}
u_{1}(k) \\
u_{2}(k) \\
\vdots \\
u_{N}(k)
\end{array}\right], \\
y(k)= & {\left[\begin{array}{c}
y_{1}(k) \\
y_{2}(k) \\
\vdots \\
y_{N}(k)
\end{array}\right], } \\
f(x(k))= & {\left[\begin{array}{c}
f_{1}(x(k)) \\
f_{2}(x(k)) \\
\vdots \\
f_{N}(x(k))
\end{array}\right], }
\end{aligned}
$$

$A=\operatorname{diag}\left(A_{i}\right), B=\operatorname{diag}\left(B_{i}\right)$ and $C=\operatorname{diag}\left(C_{i}\right)$ with $A \in$ $R^{\sum_{i=1}^{N} n_{i} \times \sum_{i=1}^{N} n_{i}}, \quad B \in R^{\sum_{i=1}^{N} n_{i} \times \sum_{i=1}^{N} m_{i}}, \quad$ and $\quad C \in R^{\sum_{i=1}^{N} p_{i} \times \Sigma_{i=1}^{N} n_{i}}$, respectively.

To consider the output tracking problem of an interconnected system (1), let the desired trajectory of the $i$ th subsystem be $r_{i}(k) \in R^{p_{i}}(i=1,2, \ldots, N)$. The following assumption is made concerning the preview ability of $r_{i}(k)$.

Assumption 2. There exists a constant vector $r_{i}$ such that $\lim _{k \longrightarrow \infty} r_{i}(k)=r_{i}$. Furthermore, $r_{i}(k)$ is previewable and the preview length is $M_{r}$; that is, at each time $k$, the current and $M_{r_{i}}$ future values $r_{i}(k), r_{i}(k+1), \ldots, r_{i}\left(k+M_{r_{i}}\right)$ of the desired trajectory are available. Additionally, the future values of the desired trajectory beyond $k+M_{r_{i}}$ are assumed to be unchanged, namely,

$$
r_{i}(k+j)=r_{i}\left(k+M_{r_{i}}\right), \quad j=M_{r_{i}}+1, M_{r_{i}}+2, \ldots
$$

Remark 2. Assumption 2 describes the previewable characteristics of the desired trajectory of the ith subsystem, and it is a rather standard hypothesis in the field of preview control [20, 26, 31, 32]. As commented in [33], the desired reference trajectories of control systems can be previewed in many applications. For example, an industrial robot manipulator usually follows a preset trajectory [30]. A humanoid robot also follows a walking pattern that can be previewed [31]. In this situation, the information of future reference trajectory can be fully utilized for the controller design to improve the tracking performance. Additionally, it should be noted that, as to an interconnected system (1), the output of each individual subsystem can track a different reference trajectory, and the desired trajectories are allowed to have different preview lengths, which is rather reasonable for practical applications.
Let the output tracking error of the ith subsystem be

$$
e_{i}(k)=y_{i}(k)-r_{i}(k)
$$

2.1. Control Objective. For system (1) (Assumptions 1 and 2 hold), find a set of observer-based decentralized preview tracking control policies $\left\{u_{1}(k), \ldots, u_{i}(k), \ldots, u_{N}(k)\right\}$ such that the output vector $y_{i}(k)$ of each subsystem asymptotically tracks the desired trajectory $r_{i}(k)$ while being subjected to nonlinear interconnected perturbations, i.e., $\lim _{k \longrightarrow+\infty} e_{i}(k)=0$ for $i=1,2, \ldots, N$.

The following lemmas play important roles in the theoretical developments.

Lemma 1 (Schur Complement lemma) (see [35]). A given matrix $S=\left[\begin{array}{ll}S_{11} & S_{12} \\ S_{12}^{T} & S_{22}\end{array}\right]<0$ is equivalent to any one of the following conditions:
(i) $S_{22}<0, S_{11}-S_{12} S_{22}^{-1} S_{12}^{T}<0$
(ii) $S_{11}<0, S_{22}-S_{12}^{T} S_{11}^{-1} S_{12}<0$

Lemma 2 (see [36]). For matrices $T, H, V$, and $W$ with appropriate dimensions and scalar $\xi$, the inequality

$$
T+W^{T} H^{T}+H W<0
$$

is fulfilled if the following condition holds:

$$
\left[\begin{array}{cc}
T & * \\
\xi H^{T}+V W & -\xi V-\xi V^{T}
\end{array}\right]<0 .
$$

Lemma 3 (see [37]). If there are matrices $P>0$ and $G$ with appropriate dimensions, then

$$
-G^{T} P^{-1} G \leq P-G-G^{T} .
$$

\section{Construction of the Augmented Error System}

The state observer of the $i$ th subsystem, which depends only on the control input and the output of the considered subsystem, can be described by

$$
\left\{\begin{array}{l}
\widehat{x}_{i}(k+1)=A_{i} \hat{x}_{i}(k)+B_{i} u_{i}(k)+L_{i}\left(y_{i}(k)-\hat{y}_{i}(k)\right), \\
\hat{y}_{i}(k)=C_{i} \hat{x}_{i}(k) \\
i=1,2, \ldots, N
\end{array}\right.
$$

where $\hat{x}_{i}(k) \in R^{n_{i}}$ is the local observed state vector and $L_{i} \in R^{n_{i} \times p_{i}}$ is the observation gain matrix of the $i$ th subsystem.

The state observer of the global system, which is composed of $N$-local observers, can be expressed as follows:

$$
\left\{\begin{array}{l}
\hat{x}(k+1)=A \hat{x}(k)+B u(k)+L(y(k)-\hat{y}(k)), \\
\hat{y}(k)=C \hat{x}(k),
\end{array}\right.
$$


where $\hat{x}(k)=\left[\begin{array}{llll}\hat{x}_{1}^{T}(k) & \hat{x}_{2}^{T}(k) & \ldots & \hat{x}_{N}^{T}(k)\end{array}\right]^{T}$ and $L=$ $\operatorname{diag}\left(L_{i}\right), i=1,2, \ldots, N$ is the block-diagonal observation gain matrix.

It should be mentioned that the state observation structure of the global interconnected nonlinear system is totally decentralized, since there is no information interchange between local state observers, thereby leading to a readily implementable and more economical scheme.

The main feature consists in developing a suitable augmented error system to transform the tracking control problem into a regulation problem. For this objective, we define the observation error between the real state and its estimation as follows:

$$
e_{x_{i}}(k)=x_{i}(k)-\widehat{x}_{i}(k) \text {. }
$$

The dynamics of the observation error $e_{x_{i}}(k)$, combing equations (1) and (10), can be described by

$$
e_{x_{i}}(k+1)=\left(A_{i}-L_{i} C_{i}\right) e_{x_{i}}(k)+f_{i}(x(k)) .
$$

In the following, the classical difference approach is used to construct the augmented subsystem of the $i$ th subsystem. We select the first-order forward difference operator:

$$
\Delta \eta(k)=\eta(k+1)-\eta(k)
$$

where $\eta(k)$ is a column vector.

From equation (6), the output equation of (1) and the definition of (14), the tracking error dynamics of the $i$ th subsystem are given by the following state representation:

$$
\begin{aligned}
e_{i}(k+1) & =e_{i}(k)+\Delta e_{i}(k) \\
& =e_{i}(k)+\Delta y_{i}(k)-\Delta r_{i}(k) \\
& =e_{i}(k)+C_{i}\left(\Delta \widehat{x}_{i}(k)+\Delta e_{x_{i}}(k)\right)-\Delta r_{i}(k) .
\end{aligned}
$$

Applying the operator $\Delta$ to the local state observer (10) yields

$$
\begin{aligned}
\Delta \hat{x}_{i}(k+1) & =A_{i} \Delta \hat{x}_{i}(k)+B_{i} \Delta u_{i}(k)+L_{i}\left(\Delta y_{i}(k)-C_{i} \Delta \widehat{x}_{i}(k)\right) \\
& =A_{i} \Delta \widehat{x}_{i}(k)+B_{i} \Delta u_{i}(k)+L_{i} C_{i}\left(\Delta x_{i}(k)-\Delta \widehat{x}_{i}(k)\right) \\
& =A_{i} \Delta \widehat{x}_{i}(k)+B_{i} \Delta u_{i}(k)+L_{i} C_{i} \Delta e_{x_{i}}(k) .
\end{aligned}
$$

To introduce the available future knowledge of the desired trajectory, we define a new vector:

$$
x_{r_{i}}(k)=\left[\begin{array}{c}
\Delta r_{i}(k) \\
\Delta r_{i}(k+1) \\
\vdots \\
\Delta r_{i}\left(k+M_{r_{i}}\right)
\end{array}\right] \in R^{p_{i}\left(M_{r_{i}}+1\right)}
$$

Note that the $M_{r_{i}}$-steps future knowledge that is available from the current time $k$ with respect to the desired trajectory $r_{i}(k)$ is summarized into vector $x_{r_{i}}(k)$. The introduction of $x_{r_{i}}(k)$ plays a significant role in preview controller design.

Under Assumption 2, the dynamics of $x_{r_{i}}(k)$ can be written as

$$
x_{r_{i}}(k+1)=A_{r_{i}} x_{r_{i}}(k)
$$

with

$$
A_{r_{i}}=\left[\begin{array}{ccccc}
0 & I_{p_{i}} & 0 & \cdots & 0 \\
0 & 0 & I_{p_{i}} & \cdots & 0 \\
\vdots & \vdots & \vdots & & \vdots \\
0 & 0 & 0 & \cdots & I_{p_{i}} \\
0 & 0 & 0 & \cdots & 0
\end{array}\right] .
$$

To consider the known future values of the desired trajectory for the controller design, we define the augmented state vector $\quad \bar{x}_{i}(k)=\left[\begin{array}{lll}e_{i}^{\mathrm{T}}(k) \Delta \hat{x}_{i}^{\mathrm{T}}(k) & x_{r_{i}}^{\mathrm{T}}(k)\end{array}\right]^{\mathrm{T}} \quad$ with $\bar{x}_{i}(k) \in R^{p_{i}+n_{i}+p_{i}\left(M_{r_{i}}+1\right)}$, and equations (15)-(18) are combined together to yield the following augmented error subsystem:

$$
\bar{x}_{i}(k+1)=\bar{A}_{i} \bar{x}_{i}(k)+\bar{B}_{i} \Delta u_{i}(k)+\bar{L}_{i} \Delta e_{x_{i}}(k),
$$

where

$$
\begin{aligned}
\bar{A}_{i} & =\left[\begin{array}{ccc}
I & C_{i} & -G_{r_{i}} \\
0 & A_{i} & 0 \\
0 & 0 & A_{r_{i}}
\end{array}\right], \\
G_{r_{i}} & =\left[\begin{array}{cccc}
I & \underbrace{0}_{p_{i} \times p_{i} M_{r_{i}}} \cdots & 0
\end{array}\right], \\
\bar{B}_{i} & =\left[\begin{array}{c}
0 \\
B_{i} \\
0
\end{array}\right], \\
\bar{L}_{i} & =\left[\begin{array}{c}
C_{i} \\
L_{i} C_{i} \\
0
\end{array}\right] .
\end{aligned}
$$

The global system composed of local systems (20) is characterized by the following representation:

$$
\bar{x}(k+1)=\bar{A} \bar{x}(k)+\bar{B} \Delta u(k)+\bar{L} \Delta e_{x}(k),
$$

where $\bar{A}=\operatorname{diag}\left(\bar{A}_{i}\right), \bar{B}=\operatorname{diag}\left(\bar{B}_{i}\right)$, and $\bar{L}=\operatorname{diag}\left(\bar{L}_{i}\right)$ with $\bar{A} \epsilon$ $R^{\sum_{i=1}^{N}\left[p_{i}+n_{i}+p_{i}\left(M_{r_{i}}+1\right)\right] \times \Sigma_{i=1}^{N}\left[p_{i}+n_{i}+p_{i}\left(M_{r_{i}}+1\right)\right]}, \bar{B} \in R^{\sum_{i=1}^{N}\left[p_{i}+n_{i}+p_{i}\left(M_{r_{i}}+1\right)\right]}$ $\times \sum_{i=1}^{N} m_{i}$, and $\bar{L} \in R^{\sum_{i=1}^{N}\left[p_{i}+n_{i}+p_{i}\left(M_{r_{i}}+1\right)\right] \times \sum_{i=1}^{N} n_{i}}$.

To analyze the dynamic behavior of the observation error equation (13) and synthesize the observer-based tracking control law with preview action, the difference operator $\Delta$ is applied to (13) to derive

$$
\Delta e_{x_{i}}(k+1)=\left(A_{i}-L_{i} C_{i}\right) \Delta e_{x_{i}}(k)+\Delta f_{i}(x(k)),
$$

with $\Delta f_{i}(x(k))=f_{i}(x(k+1))-f_{i}(x(k))$.

The global system of (23) is described by

$$
\Delta e_{x}(k+1)=(A-L C) \Delta e_{x}(k)+\Delta f(x(k)),
$$


where $A, L$, and $C$ are defined as before, and

$$
\Delta f(x)=\left[\begin{array}{c}
\Delta f_{1}(x) \\
\Delta f_{2}(x) \\
\vdots \\
\Delta f_{N}(x)
\end{array}\right] .
$$

By combining equations (22) and (24) together, the required augmented error system is derived as follows:

$$
\left\{\begin{array}{l}
\bar{x}(k+1)=\bar{A} \bar{x}(k)+\bar{B} \Delta u(k)+\bar{L} \Delta e_{x}(k), \\
\Delta e_{x}(k+1)=(A-L C) \Delta e_{x}(k)+\Delta f(x(k)) .
\end{array}\right.
$$

If we find a suitable controller to ensure that the closedloop system of system (26) is asymptotically stable, then the expected output tracking is achieved, since the tracking error is a component of the state vector $\bar{x}(k)$. Therefore, the output tracking problem under consideration is reduced to a stabilization problem of system (26).

\section{Observer-Based Decentralized Preview Tracking Controller Design}

As for the $i$ th augmented subsystem in (20) and (23), we introduce the state feedback controller

$$
\Delta u_{i}(k)=K_{i} \bar{x}_{i}(k),
$$

where $K_{i} \in R^{m_{i} \times\left[p_{i}+n_{i}+p_{i}\left(M_{r_{i}}+1\right)\right]}$ is the controller gain matrix of the $i$ th augmented subsystem to be determined.

The control law of the global system (26) is given in the following form:

$$
u(k)=K \bar{x}(k),
$$

where $K=\operatorname{diag}\left(K_{i}\right), i=1,2, \ldots, N$ is the block-diagonal controller gain matrix.

The closed-loop system of the augmented system (26), applying the control law (28), is given by

$$
\left\{\begin{array}{l}
\bar{x}(k+1)=(\bar{A}+\bar{B} K) \bar{x}(k)+\bar{L} \Delta e_{x}(k), \\
\Delta e_{x}(k+1)=(A-L C) \Delta e_{x}(k)+\Delta f(x(k)) .
\end{array}\right.
$$

To effectively compute the decentralized control gain matrix $K=\operatorname{diag}\left(K_{i}\right)$ and the observation gain matrix $L=\operatorname{diag}\left(L_{i}\right)$, in the following, an innovative design approach for the asymptotic stability of the overall closed-loop system (29) is presented.

Theorem 1. Suppose that Assumptions 1 and 2 hold. Given scalar $\xi>0$, if there are matrices $P>0, S>0, G_{1}, G_{2}, X, U$, and $V$ such that

$$
\left[\begin{array}{cccccccc}
\Upsilon_{11} & * & * & * & * & * & * & * \\
0 & \Upsilon_{22} & * & * & * & * & * & * \\
0 & 0 & -I & * & * & * & * & * \\
\Upsilon_{41} & \Upsilon_{42} & 0 & -P & * & * & * & * \\
0 & \Upsilon_{52} & I & 0 & -S & * & * & * \\
F_{x} G_{1} & 0 & 0 & 0 & 0 & -\eta I & * & * \\
0 & G_{2} & 0 & 0 & 0 & 0 & -\eta I & * \\
0 & \Upsilon_{82} & 0 & \Upsilon_{84} & -\xi U^{T} & 0 & 0 & \Upsilon_{88}
\end{array}\right]<0
$$

where

$$
\begin{aligned}
& \Upsilon_{11}=P-G_{1}-G_{1}^{T}, \\
& \Upsilon_{41}=\bar{A} G_{1}+\bar{B} X, \\
& \Upsilon_{22}=S-G_{2}-G_{2}^{T}, \\
& \Upsilon_{42}=T_{e} C G_{2}+T_{s} U C, \\
& \Upsilon_{52}=A G_{2}-U C, \\
& \Upsilon_{82}=C G_{2}-V C, \\
& \Upsilon_{84}=\xi U^{T} T_{s}^{T}, \\
& \Upsilon_{88}=-\xi V-\xi V^{T}, \\
& \eta=\frac{1}{\sum_{i=1}^{N} \gamma_{i}^{2}},
\end{aligned}
$$

and $F_{x}, T_{e}$, and $T_{s}$ are given by (36), (47), and (48), respectively, then the closed-loop system (29) is globally asymptotically stable. Furthermore, the decentralized controller gain $K=\operatorname{diag}\left(K_{i}\right)$ and the decentralized observer gain $L=$ $\operatorname{diag}\left(L_{i}\right)$ are derived by $K=X G_{1}^{-1}$ and $L=U V^{-1}$, respectively.

Proof: Consider the Lyapunov function

$$
\begin{aligned}
V\left(\bar{x}, \Delta e_{x}\right) & =\left[\begin{array}{ll}
\bar{x}^{T} & \Delta e_{x}^{T}
\end{array}\right]\left[\begin{array}{cc}
P^{-1} & 0 \\
0 & S^{-1}
\end{array}\right]\left[\begin{array}{c}
\bar{x} \\
\Delta e_{x}
\end{array}\right] \\
& =\bar{x}^{T} P^{-1} \bar{x}+\Delta e_{x}^{T} S^{-1} \Delta e_{x},
\end{aligned}
$$

where $P=\operatorname{diag}\left(P_{i}\right)>0 \quad$ and $\quad S=\operatorname{diag}\left(S_{i}\right)>0 \quad$ with $P_{i} \in R^{\left[p_{i}+n_{i}+p_{i}\left(M_{r_{i}}+1\right)\right] \times\left[p_{i}+n_{i}+p_{i}\left(M_{r_{i}}+1\right)\right]}$ and $S_{i} \in R^{n_{i} \times n_{i}}$, respectively. Thus, the function $V$ is positive-definite. Taking its difference along the trajectory of (29) leads to 


$$
\begin{aligned}
\Delta V\left(\bar{x}, \Delta e_{x}\right)= & \bar{x}^{T}(k+1) P^{-1} \bar{x}(k+1)+\Delta e_{x}^{T}(k+1) S^{-1} \Delta e_{x}(k+1)-\bar{x}^{T}(k) P^{-1} \bar{x}(k)-\Delta e_{x}^{T}(k) S^{-1} \Delta e_{x}(k) \\
= & \left((\bar{A}+\bar{B} K) \bar{x}(k)+\bar{L} \Delta e_{x}(k)\right)^{T} P^{-1}\left((\bar{A}+\bar{B} K) \bar{x}(k)+\bar{L} \Delta e_{x}(k)\right)+\left((A-L C) \Delta e_{x}(k)+\Delta f(x(k))\right)^{T} S^{-1} \\
& \cdot\left((A-L C) \Delta e_{x}(k)+\Delta f(x(k))\right)-\bar{x}^{T}(k) P^{-1} \bar{x}(k)-\Delta e_{x}^{T}(k) S^{-1} \Delta e_{x}(k) \\
= & \bar{x}^{T}(k)\left[(\bar{A}+\bar{B} K)^{T} P^{-1}(\bar{A}+\bar{B} K)-P^{-1}\right] \bar{x}(k)+2 \bar{x}^{T}(k)(\bar{A}+\bar{B} K)^{T} P^{-1} \bar{L} \Delta e_{x}(k)+\Delta e_{x}^{T}(k) \bar{L}^{T} P^{-1} \bar{L} \Delta e_{x}(k)+\Delta e_{x}^{T}(k) \\
& \cdot\left[(A-L C)^{T} S^{-1}(A-L C)-S^{-1}\right] \Delta e_{x}(k)+2 \Delta e_{x}^{T}(k)(A-L C)^{T} S^{-1} \Delta f(x)+\Delta f^{T}(x(k)) S^{-1} \Delta f(x(k)) .
\end{aligned}
$$

Notice that, by Assumption 1, it follows that

$$
\gamma_{i} \Delta x^{T}(k) \Delta x(k)-\Delta f_{i}^{T}(x) \Delta f_{i}(x) \geq 0
$$

$$
\Delta x(k)=\Delta \widehat{x}(k)+\Delta e_{x}(k) \triangleq F_{x} \bar{x}(k)+\Delta e_{x}(k),
$$

where

Furthermore, from equation (12), $\Delta x(k)$ can be computed by

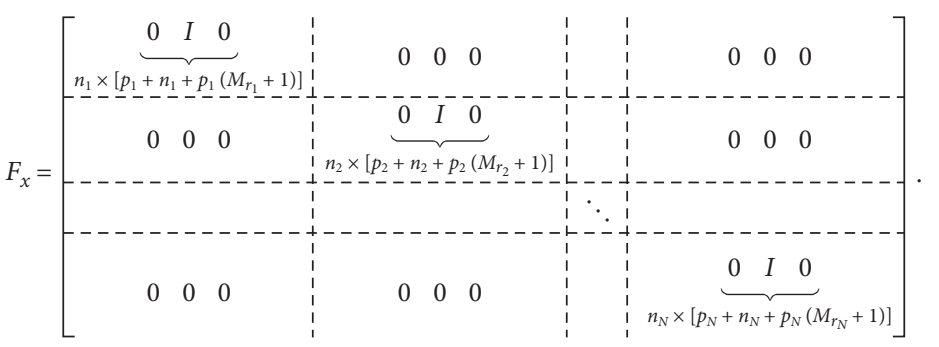

Thus, equation (34) can be rewritten as

$$
\begin{aligned}
& \gamma_{i}^{2}\left(F_{x} \bar{x}(k)+\Delta e_{x}(k)\right)^{T}\left(F_{x} \bar{x}(k)+\Delta e_{x}(k)\right) \\
& -\Delta f_{i}^{T}(x) \Delta f_{i}(x) \geq 0,
\end{aligned}
$$

that is,

$$
\begin{aligned}
& \gamma_{i}^{2}\left[\bar{x}^{T}(k) F_{x}^{T} F_{x} \bar{x}(k)+2 \bar{x}^{T}(k) F_{x}^{T} \Delta e_{x}(k)\right. \\
& \left.\quad+\Delta e_{x}^{T}(k) \Delta e_{x}(k)\right]-\Delta f_{i}^{T}(x) \Delta f_{i}(x) \geq 0 .
\end{aligned}
$$

Summing both sides of the above inequality from $i=1$ to $N$ results in

$$
\begin{aligned}
\sum_{i=1}^{N} \gamma_{i}^{2} & {\left[\bar{x}^{T}(k) F_{x}^{T} F_{x} \bar{x}(k)+2 \bar{x}^{T}(k) F_{x}^{T} \Delta e_{x}(k)+\Delta e_{x}^{T}(k) \Delta e_{x}(k)\right] } \\
& -\sum_{i=1}^{N} \Delta f_{i}^{T}(x) \Delta f_{i}(x) \\
= & \sum_{i=1}^{N} \gamma_{i}^{2}\left[\bar{x}^{T}(k) F_{x}^{T} F_{x} \bar{x}(k)+2 \bar{x}^{T}(k) F_{x}^{T} \Delta e_{x}(k)\right. \\
& \left.+\Delta e_{x}^{T}(k) \Delta e_{x}(k)\right]-\Delta f^{T}(x) \Delta f(x) \geq 0 .
\end{aligned}
$$

$$
\begin{aligned}
\Delta V\left(\bar{x}, \Delta e_{x}\right) \leq & \bar{x}^{T}(k)\left[(\bar{A}+\bar{B} K)^{T} P^{-1}(\bar{A}+\bar{B} K)-P^{-1}\right] \bar{x}(k) \\
& +2 \bar{x}^{T}(k)(\bar{A}+\bar{B} K)^{T} P^{-1} \bar{L} \Delta e_{x}(k) \\
& +\Delta e_{x}^{T}(k) \bar{L}^{T} P^{-1} \bar{L} \Delta e_{x}(k)+\Delta e_{x}^{T}(k) \\
& \cdot\left[(A-L C)^{T} S^{-1}(A-L C)-S^{-1}\right] \Delta e_{x}(k) \\
& +2 \Delta e_{x}^{T}(k)(A-L C)^{T} S^{-1} \Delta f(x) \\
& +\Delta f^{T}(x(k)) S^{-1} \Delta f(x(k)) \\
& +\sum_{i=1}^{N} \gamma_{i}^{2}\left[\bar{x}^{T}(k) F_{x}^{T} F_{x} \bar{x}(k)\right. \\
& \left.+2 \bar{x}^{T}(k) F_{x}^{T} \Delta e_{x}(k)+\Delta e_{x}^{T}(k) \Delta e_{x}(k)\right] \\
& -\Delta f^{T}(x) \Delta f(x) \\
= & {\left[\bar{x}^{T}(k) \Delta e_{x}^{T}(k) \Delta f^{T}(x(k))\right] } \\
& \cdot \Xi\left[\bar{x}^{T}(k) \Delta e_{x}^{T}(k) \Delta f^{T}(x(k))\right]^{T},
\end{aligned}
$$

where

$$
\Xi=\left[\begin{array}{ccc}
\Xi_{11} & * & * \\
\Xi_{21} & \Xi_{22} & * \\
0 & \Xi_{32} & \Xi_{33}
\end{array}\right],
$$

Thus, from (39), $\Delta V$ is further bounded by 
with

$$
\begin{aligned}
\Xi_{11} & =(\bar{A}+\bar{B} K)^{T} P^{-1}(\bar{A}+\bar{B} K)-P^{-1}+\eta^{-1} F_{x}^{T} F_{x}, \\
\Xi_{21} & =\bar{L}^{T} P^{-1}(\bar{A}+\bar{B} K)+\eta^{-1} F_{x}, \\
\Xi_{22} & =\bar{L}^{T} P^{-1} \bar{L}+(A-L C)^{T} S^{-1}(A-L C)-S^{-1}+\eta^{-1} I, \\
\eta & =\frac{1}{\sum_{i=1}^{N} \gamma_{i}^{2}}, \\
\Xi_{32} & =S^{-1}(A-L C), \\
\Xi_{33} & =S^{-1}-I .
\end{aligned}
$$

Therefore, if the inequality $\Gamma<0$ is satisfied, then $\Delta V<0$ holds for all $\left[\bar{x}^{T}(k) \Delta e_{x}^{T}(k)\right]^{T} \neq 0$. Based on the Lyapunov stability theorem, the closed-loop system (29) is globally asymptotically stable at the origin.

Applying the Schur complement lemma (i.e., Lemma 1), $\Gamma<0$ in equation (40) is equivalent to

$$
\left[\begin{array}{ccccccc}
-P^{-1} & * & * & * & * & * & * \\
0 & -S^{-1} & * & * & * & * & * \\
0 & 0 & -I & * & * & * & * \\
\bar{A}+\bar{B} K & \bar{L} & 0 & -P & * & * & * \\
0 & A-L C & I & 0 & -S & * & * \\
F_{x} & 0 & 0 & 0 & 0 & -\eta I & * \\
0 & I & 0 & 0 & 0 & 0 & -\eta I
\end{array}\right]<0 .
$$

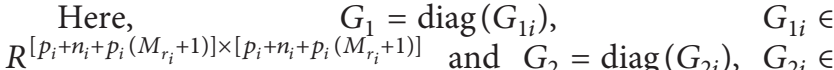
$R^{n_{i} \times n_{i}}$ have been used.

By using the $\bar{L}_{i}$ defined in (20), the element $\bar{L} G_{2}$ that appears in (44) is given by

$$
\begin{aligned}
\bar{L} G_{2} & =\operatorname{diag}\left(\bar{L}_{i}\right) \times \operatorname{diag}\left(G_{2 i}\right) \\
& =\operatorname{diag}\left(\left[\begin{array}{c}
C_{i} \\
L_{i} C_{i} \\
0
\end{array}\right]\right) \times \operatorname{diag}\left(G_{2 i}\right) \\
& =\operatorname{diag}\left(\left[\begin{array}{c}
C_{i} G_{2 i} \\
L_{i} C_{i} G_{2 i} \\
0
\end{array}\right]\right) .
\end{aligned}
$$

In view of Lemma 3, inequality (44) holds if

$$
\left[\begin{array}{ccccccc}
P-G_{1}-G_{1}^{T} & * & * & * & * & * & * \\
0 & S-G_{2}-G_{2}^{T} & * & * & * & * & * \\
0 & 0 & -I & * & * & * & * \\
(\bar{A}+\bar{B} K) G_{1} & \operatorname{diag}\left(\left[\begin{array}{c}
C_{i} G_{2 i} \\
L_{i} C_{i} G_{2 i} \\
0
\end{array}\right]\right) & 0 & -P & * & * & * \\
0 & A G_{2}-L C G_{2} & I & 0 & -S & * & * \\
F_{x} G_{1} & 0 & 0 & 0 & 0 & -\eta I & * \\
0 & G_{2} & 0 & 0 & 0 & 0 & -\eta I
\end{array}\right]<0 .
$$


By some mathematical operations, after denoting $p=\sum_{i=1}^{N} p_{i}, n=\sum_{i=1}^{N} n_{i}$, we obtain

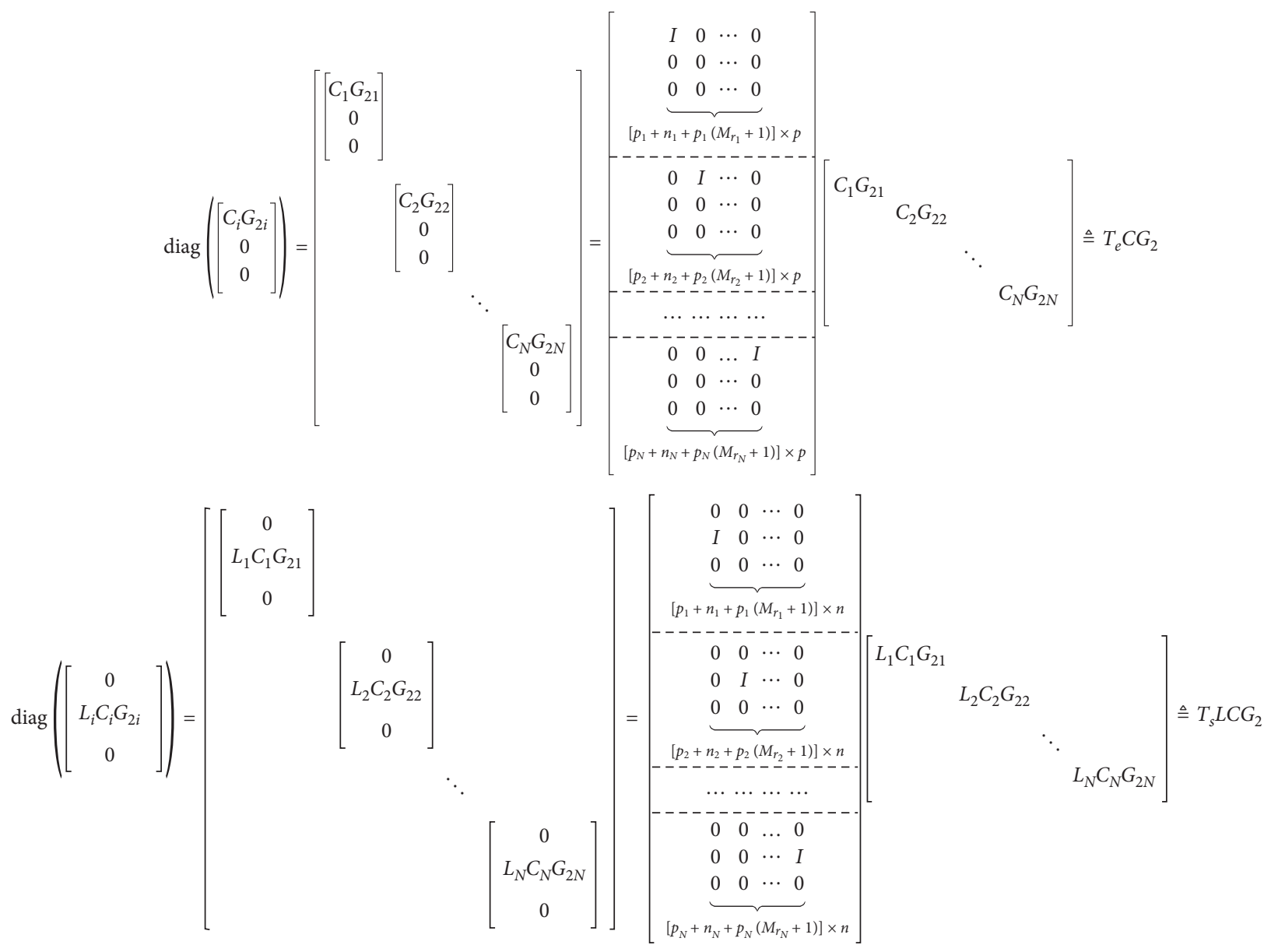
that

Thus, from the above equations (47) and (48), it follows

$$
\begin{aligned}
\operatorname{diag}\left(\left[\begin{array}{c}
C_{i} G_{2 i} \\
L_{i} C_{i} G_{2 i} \\
0
\end{array}\right]\right) & =\operatorname{diag}\left(\left[\begin{array}{c}
C_{i} G_{2 i} \\
0 \\
0
\end{array}\right]\right)+\operatorname{diag}\left(\left[\begin{array}{c}
0 \\
L_{i} C_{i} G_{2 i} \\
0
\end{array}\right]\right) \\
& =T_{e} C G_{2}+T_{s} L C G_{2} .
\end{aligned}
$$

Hence, inequality (46) becomes

$$
\left[\begin{array}{ccccccc}
P-G_{1}-G_{1}^{T} & * & * & * & * & * & * \\
0 & S-G_{2}-G_{2}^{T} & * & * & * & * & * \\
0 & 0 & -I & * & * & * & * \\
(\bar{A}+\bar{B} K) G_{1} & T_{e} C G_{2}+T_{s} L C G_{2} & 0 & -P & * & * & * \\
0 & A G_{2}-L C G_{2} & I & 0 & -S & * & * \\
F_{x} G_{1} & 0 & 0 & 0 & 0 & -\eta I & * \\
0 & G_{2} & 0 & 0 & 0 & 0 & -\eta I
\end{array}\right]<0 .
$$

The matrix in the left side of (50) is denoted by $\Phi$. With the change of variable $X=K G_{1}$, the controller gain matrix is determined by $K=X G_{1}^{-1}$. Since $X=\operatorname{diag}\left(X_{i}\right)$, the local controller gain is $K_{i}=X_{i} G_{1 i}^{-1}$ for $i=1,2, \ldots, N$. To effectively compute the observation gain, let us introduce auxiliary invertible matrices $V_{i}(i=1,2, \ldots, N)$ and define $L_{i}=U_{i} V_{i}^{-1}$. Then, the following equation holds:

$$
L_{i} C_{i} G_{2 i}=U_{i} V_{i}^{-1}\left(C_{i} G_{2 i}-V_{i} C_{i}\right)+U_{i} C_{i} .
$$

Furthermore, let $U=\operatorname{diag}\left(U_{i}\right)$ and $V=\operatorname{diag}\left(V_{i}\right)$. Then, the element $L C G_{2}$ in (50) is given by

$$
\begin{aligned}
L C G_{2} & =\operatorname{diag}\left(L_{i} C_{i} G_{2 i}\right) \\
& =\operatorname{diag}\left(U_{i} V_{i}^{-1}\left(C_{i} G_{2 i}-V_{i} C_{i}\right)+U_{i} C_{i}\right) \\
& =U V^{-1}\left(C G_{2}-V C\right)+U C .
\end{aligned}
$$

Using the variable substitution with (52), the matrix $\Phi$ on the left side of inequality (50) can be expressed in the form of 


$$
\Phi=\Sigma_{1}+\operatorname{sym}(\underbrace{\left[\begin{array}{c}
0 \\
0 \\
0 \\
T_{s} U \\
-U \\
0 \\
0
\end{array}\right]}_{H} \underbrace{V^{-1}\left[\begin{array}{llllllll}
0 & C G_{2}-V C & 0 & 0 & 0 & 0 & 0
\end{array}\right]}_{W}),
$$

where

$$
\Sigma_{1}=\left[\begin{array}{ccccccc}
P-G_{1}-G_{1}^{T} & * & * & * & * & * & * \\
0 & S-G_{2}-G_{2}^{T} & * & * & * & * & * \\
0 & 0 & -I & * & * & * & * \\
\bar{A} G_{1}+\bar{B} X & T_{e} C G_{2}+T_{s} U C & 0 & -P & * & * & * \\
0 & A G_{2}-U C & I & 0 & -S & * & * \\
F_{x} G_{1} & 0 & 0 & 0 & 0 & -\eta I & * \\
0 & G_{2} & 0 & 0 & 0 & 0 & -\eta I
\end{array}\right] .
$$

According to Lemma 1 , the inequality $\Phi<0$ can be guaranteed by the following condition:

$$
\left[\begin{array}{cc}
\Sigma_{1} & * \\
\Sigma_{2} & -\xi V-\xi V^{T}
\end{array}\right]<0
$$

where

$$
\Sigma_{2}=\xi H^{T}+V W=\left[\begin{array}{lllllll}
0 & C G_{2}-V C & 0 & \xi U^{T} T_{s}^{T} & -\xi U^{T} & 0 & 0
\end{array}\right] .
$$

The inequality (55) can be formulated in the form of (30). Therefore, if condition (30) is satisfied, then condition (50) holds. Furthermore, $\Gamma<0$ is ensured.

Remark 3. It should be noted that Theorem 1 provides a new criterion to ensure the asymptotic stability of the overall closed-loop system (29) in terms of LMI formulations. With the help of the LMI toolbox in MATLAB, both the decentralized observer gain $L=\operatorname{diag}\left(L_{i}\right)$ and the decentralized controller gain $K=\operatorname{diag}\left(K_{i}\right)$ can be computed simultaneously. It can be noticed that the matrix variables $L$ and $G_{2}$ in (50) appear in a nonlinear coupling form, which results in some computation troubles for the observer design. To overcome this obstacle, the auxiliary matrix variables $U=\operatorname{diag}\left(U_{i}\right)$ and $V=\operatorname{diag}\left(V_{i}\right)$ in the form of (52) are introduced. As a result, the decentralized observer-based decentralized preview tracking controller can be resolved through a one-step procedure. Moreover, with the aid of additional matrices $G_{i}(i=1,2)$, the slack variable method $[38,39]$ is used to derive the LMI-based relaxation condition.
Additionally, our adopted design method can generalize the results in $[7,11,40,41]$ and can be effectively applied to the $H_{\infty}$ model reference decentralized tracking control of the interconnected systems in discrete-time domain.

Remark 4. It should be mentioned that, in Assumption 1, the specific values for the interconnection bounds $\gamma_{i}$ are set according to the nonlinear interconnection term $f_{i}(x)$. As a matter of fact, our obtained results can also be applied to the case where the parameter $\gamma_{i}$ is unknown and $f_{i}(x)$ may be uncertain. Inspired by $[7,40]$, in this situation, the parameter bounds of Lipschitz interconnected terms $\gamma_{i}(i=1,2, \ldots, N)$ can be maximized and the overall closed-loop system remains asymptotically stable, whereas the parameters $\eta_{i}(i=$ $1,2, \ldots, N)$ are minimized as much as possible. As a result, the decentralized observer-based decentralized tracking controller design can be characterized by the following optimization problem:

$$
\left\{\begin{array}{l}
\text { minimize } \eta \\
\text { subject to } P>0, S>0, G_{1}, G_{2}, X, U, V \text { and (30). }
\end{array}\right.
$$

By solving this minimization problem, the maximized values of the Lipschitz connection bounds can be found so that the control scheme may be applied to a larger nonlinear coverage.

When the LMI feasibility problem in Theorem 1 is solvable, the controller gain in (28) for the global augmented error system (26) is determined by $K=X G_{1}^{-1}$. Correspondingly, the local controller gain is obtained by $K_{i}=$ $X_{i} G_{1 i}^{-1}$ for each local subsystem. To simplify the expression and to clarify the control structure of the proposed observerbased decentralized tracking controller with preview action, the local controller gain $K_{i}$ is partitioned as

$$
\begin{aligned}
K_{i} & =\left[\begin{array}{lllll}
K_{e_{i}} & K_{\widehat{x}_{i}} K_{r_{i}}
\end{array}\right] \\
& =\left[\begin{array}{lllll}
K_{e_{i}}: K_{\widehat{x}_{i}}: k_{r_{i}}(0) & k_{r_{i}}(1) & \ldots & k_{r_{i}}\left(M_{r_{i}}\right)
\end{array}\right] .
\end{aligned}
$$

Hence, the local controller (27) can be expressed by

$$
\Delta u_{i}(k)=K_{e_{i}} e_{i}(k)+K_{\widehat{x}_{i}} \Delta \widehat{x}_{i}(k)+K_{r_{i}} x_{r_{i}}(k) .
$$

By solving the control input $u_{i}(k)$ from the above equation, we obtain

$$
\begin{aligned}
u_{i}(k) & =\sum_{j=-M_{r_{i}}-1}^{k-1} \Delta u_{i}(j)+u\left(-M_{r_{i}}-1\right) \\
& =K_{e_{i}} \sum_{j=0}^{k-1} e_{i}(j)+K_{\widehat{x}_{i}} \widehat{x}_{i}(k)+K_{r_{i}} x_{r_{i}}(k) .
\end{aligned}
$$

Here, the assumption that $\widehat{x}_{i}(j)=0, u_{i}(j)=0$ and $r_{i}(j)=0$ for $j<0$ has been used.

Theorem 2. Suppose that Assumptions 1 and 2 hold. If the LMI condition (30) in Theorem 1 is feasible, then the observerbased decentralized preview controller for system (1) is 


$$
\begin{aligned}
& u(k)=\left[\begin{array}{llll}
u_{1}^{T}(k) & u_{2}^{T}(k) & \cdots & u_{N}^{T}(k)
\end{array}\right]^{T}, \\
& u_{i}(k)=K_{e_{i}} \sum_{j=0}^{k-1} e_{i}(j)+K_{\widehat{x}_{i}} \widehat{x}_{i}(k)+\sum_{j=0}^{M_{r_{i}}} k_{r_{i}}(j) r_{i}(k+j), \\
& \\
&(i=1,2, \ldots, N),
\end{aligned}
$$

where $\hat{x}_{i}(k)$ is given by (10) with the observer gain $L_{i}=U_{i} V_{i}^{-1}$ $(i=1,2, \ldots, N)$, and the controller gains $K_{e_{i}} K_{\hat{x}_{i}}$, and $k_{r_{i}}(j)\left(j=0,1, \ldots, M_{r_{i}}\right)$ are determined by (58). Using this controller, the output vector $y_{i}(k)$ can asymptotically track the desired trajectory $r_{i}(k)$ for $i=1,2, \ldots, N$.

Remark 5. The observer-based preview tracking control law proposed in this paper has a completely decentralized structure, as shown in (61), since there is no information transfer between local individual controllers. Furthermore, it can be seen that the control law of each subsystem consists of three terms. More specifically, the first term, $K_{e_{i}} \sum_{j=0}^{k-1} e_{i}(j)$, stands for the integral control action that is capable of eliminating the static error of the system, the second term, $K_{\widehat{x}_{i}} \hat{x}(k)$, is the observer-based state feedback control action, and the last one, $\sum_{j=0}^{M_{r_{i}}} k_{r_{i}}(j) r_{i}(k+j)$, refers to the preview compensation action of the desired trajectory that is conducive to improving the tracking performance of the overall interconnected system.
Remark 6. So far, there have been many interesting results concerning decentralized tracking control in the literature, such as $[5,9,12,39,42-47]$. Note that most of them are developed via backstepping control, adaptive dynamic programming, iterative learning control, etc. However, in this paper, for the first time, we propose a novel and effective decentralized tracking controller design methodology based on the preview control technique, which guarantees that even in the presence of Lipschitz nonlinear interconnections, all tracking errors asymptotically converge to zero. In contrast to $[6,8,23,24,26,27,29,43,44]$, where all the state variables are required to be measurable, in this paper, a more complex and challenging problem, i.e., the observer-based decentralized preview tracking control problem, is considered and the merits of the developed design method consist in two aspects: (i) an efficient preview compensation mechanism, which is obtained by integrating the known future information of the desired trajectory, is included in the proposed tracking control structure in order to improve the tracking performance; (ii) only the local information is utilized in the design procedure for each local subsystem, which is more economical and straightforward for practical applications.

\section{Numerical Simulations}

Example 1. Consider an interconnected system described by

$$
\begin{aligned}
& S_{1}:\left\{\begin{array}{l}
x_{1}(k+1)=\underbrace{\left[\begin{array}{ccc}
0 & 0.352 & 0 \\
0 & 0.145 & 0.486 \\
0.031 & -0.142 & 0.565
\end{array}\right]}_{A_{1}} x_{1}(k)+\underbrace{\left[\begin{array}{c}
1.251 \\
0.55 \\
0.302
\end{array}\right]}_{B_{1}} u_{1}(k)+\underbrace{\left[\begin{array}{c}
0.1 \sin \left(x_{11}(k)\right) \\
0.1 \cos \left(x_{22}(k)\right) \\
0
\end{array}\right]}_{f_{1}\left(x_{1}(k), x_{2}(k)\right)}, \\
y_{1}(k)=\underbrace{\left[\begin{array}{lll}
1 & 1 & 0
\end{array}\right]}_{C_{1}} x_{1}(k),
\end{array}\right. \\
& S_{2}:\left\{\begin{array}{l}
x_{2}(k+1)=\underbrace{\left[\begin{array}{cc}
0.713 & -0.965 \\
0.812 & 0.32
\end{array}\right]}_{A_{2}} x_{2}(k)+\underbrace{\left[\begin{array}{c}
0.701 \\
1.52
\end{array}\right]}_{B_{2}} u_{2}(k)+\underbrace{\left[\begin{array}{c}
0.1 \arctan \left(x_{11}(k)\right) \\
0.25 \sin \left(x_{22}(k)\right)
\end{array}\right]}_{f_{2}\left(x_{1}(k), x_{2}(k)\right)}, \\
y_{2}(k)=\underbrace{[1-1]}_{C_{2}} x_{2}(k),
\end{array}\right.
\end{aligned}
$$

where $x_{1}(k)=\left[\begin{array}{lll}x_{11}(k) & x_{12}(k) & x_{13}(k)\end{array}\right]^{T} \in R^{3}$ and $x_{2}(k)=$ $\left[x_{21}(k) x_{22}(k)\right]^{T} \in R^{2}$ are states, $y_{1}(k), y_{2}(k) \in R$ are outputs and $u_{1}(k), u_{2}(k) \in R$ are the control inputs. It is straightforward to compute that the nonlinear interconnections $f_{i}\left(x_{1}, x_{2}\right) \quad(i=1,2)$ satisfy Assumption 1 with Lipschitz constants $\gamma_{1}=0.1$ and $\gamma_{2}=0.25$.

For the purpose of the simulation, the desired trajectories are set as

$$
\begin{aligned}
& r_{1}(k)= \begin{cases}0, & k<10, \\
0.15(k-10), & 10 \leq k \leq 30, \\
3, & k>30,\end{cases} \\
& r_{2}(k)= \begin{cases}0, & k<20, \\
3, & k \geq 20 .\end{cases}
\end{aligned}
$$


Here, we assume that the desired signals $r_{1}(k)$ and $r_{2}(k)$ are both previewable, namely, Assumption 2 is satisfied. Furthermore, their preview lengths are assumed to be $M_{r_{1}}$ and $M_{r_{2}}$, respectively.

To compare the effect of the preview action of the desired trajectory on the output tracking performance, we consider three cases for the above interconnected nonlinear system, namely, (i) $M_{r_{1}}=0, M_{r_{2}}=0$, (ii) $M_{r_{1}}=2, M_{r_{2}}=3$, and (iii) $M_{r_{1}}=4, M_{r_{2}}=6$. Given $\xi=1$, the LMI feasibility problem (30) in Theorem 1 can be solved using the MATLAB LMI toolbox, and furthermore, the solutions with respect to the designed observer gain and controller gain are derived as follows:

Case 1. When $M_{r_{1}}=0, M_{r_{2}}=0$, the decentralized observer gain and decentralized controller gain are

$$
\begin{aligned}
L_{1} & =\left[\begin{array}{l}
0.1709 \\
0.0501 \\
0.0295
\end{array}\right], \\
L_{2} & =\left[\begin{array}{l}
0.6506 \\
0.2055
\end{array}\right], \\
K_{e_{1}} & =-0.0502, \\
K_{e_{2}} & =0.0258, \\
K_{\widehat{x}_{1}} & =\left[\begin{array}{ll}
-0.0565 & -0.2834-0.2523
\end{array}\right], \\
K_{\widehat{x}_{2}} & =\left[\begin{array}{ll}
-0.5032 & -0.2594
\end{array}\right] .
\end{aligned}
$$

Case 2. When $M_{r_{1}}=2, M_{r_{2}}=3$, the decentralized observer gain and decentralized controller gain are

$$
\begin{aligned}
& L_{1}=\left[\begin{array}{l}
0.1736 \\
0.0515 \\
0.0303
\end{array}\right] \text {, } \\
& L_{2}=\left[\begin{array}{l}
0.6634 \\
0.1976
\end{array}\right] \text {, } \\
& K_{e_{1}}=-0.0585 \text {, } \\
& K_{e_{2}}=0.0311 \text {, } \\
& K_{\widehat{x}_{1}}=\left[\begin{array}{lll}
-0.0647 & -0.2918 & -0.2536
\end{array}\right] \text {, } \\
& K_{\widehat{x}_{2}}=\left[\begin{array}{ll}
-0.4823 & -0.3144
\end{array}\right] \text {, } \\
& K_{r_{1}}=\left[\begin{array}{lll}
0.0585 & 0.0583 & 0.0551
\end{array}\right] \text {, } \\
& K_{r_{2}}=\left[\begin{array}{llll}
-0.0312 & -0.0311 & -0.0341 & -0.0319
\end{array}\right] \text {. }
\end{aligned}
$$

Case 3. When $M_{r_{1}}=4, M_{r_{2}}=6$, the decentralized observer gain and decentralized controller gain are

$$
\begin{aligned}
& L_{1}=\left[\begin{array}{l}
0.1765 \\
0.0504 \\
0.0316
\end{array}\right], \\
& L_{2}=\left[\begin{array}{l}
0.6733 \\
0.1987
\end{array}\right] \\
& K_{e_{1}}=-0.0721 \text {, } \\
& K_{e_{2}}=0.0383 \text {, } \\
& K_{\widehat{x}_{1}}=\left[\begin{array}{lll}
-0.0783 & -0.3060 & -0.2553
\end{array}\right] \text {, } \\
& K_{\widehat{x}_{2}}=\left[\begin{array}{ll}
-0.4672 & -0.3485
\end{array}\right] \text {, } \\
& K_{r_{1}}=\left[\begin{array}{lllll}
0.0720 & 0.0716 & 0.0658 & 0.0624 & 0.0592
\end{array}\right] \text {, } \\
& K_{r_{2}}=\left[\begin{array}{llll}
-0.0383 & -0.0386 & -0.0417 & -0.0387
\end{array}\right. \\
& -0.0354-0.0325-0.0295] \text {. }
\end{aligned}
$$

Based on Theorem 2, for the predefined reference trajectories and the assumed preview lengths, the observerbased decentralized tracking control pair $\left\{u_{1}(k), u_{2}(k)\right\}$ can be derived in the form of (58), which can guarantee the asymptotic tracking of the desired trajectory for each subsystem while being subjected to nonlinear interconnected perturbations. In the following, the closed-loop output response, the tracking error, and the control input of subsystem $S_{1}$ are shown in Figures 1-3, respectively. The corresponding simulation results for subsystem $S_{2}$ are displayed in Figures 4-6, respectively. In the numerical simulation, the initial states of the subsystems are $x_{1}=\left[\begin{array}{lll}0 & 0 & 0\end{array}\right]^{T}$ and $x_{2}=\left[\begin{array}{ll}0 & 0\end{array}\right]^{T}$, and the initial states of the observers are $\hat{x}_{1}=\left[\begin{array}{lll}0.1 & 0.2 & 0\end{array}\right]^{T}$ and $\hat{x}_{2}=\left[\begin{array}{ll}-0.1 & 0\end{array}\right]^{T}$.

From these figures, we can see that the observer-based decentralized tracking controller proposed in this paper is very effective for the expected output tracking, no matter whether the preview action exists or not. Figures 1 and 4 illustrate that the output vectors in all situations asymptotically track the desired trajectories. Compared to the conventional controller without preview action (i.e., $M_{r_{1}}=M_{r_{2}}=0$ ), the suggested decentralized tracking control law with preview action makes the system output produce faster responses and approach the desired value more quickly. The tracking error, as a good tool to evaluate the output tracking quality, is depicted in Figures 2 and 5, which indicates that the tracking error between the system output and the desired trajectory decreases dramatically as the preview length increases. Obviously, the developed preview control scheme improves the tracking performance of the overall system in spite of that the nonlinear interconnection perturbation affecting the subsystems. Furthermore, as Figures 3 and 6 show, the control signals remain bounded with a reasonable amplitude, thereby demonstrating the efficiency of the suggested control approach. Additionally, the integral error performance criteria, such as IAE, ISE, ITAE, and ITSE, are good tools to evaluate the tracking quality and are shown in Tables 1 and 2, where 


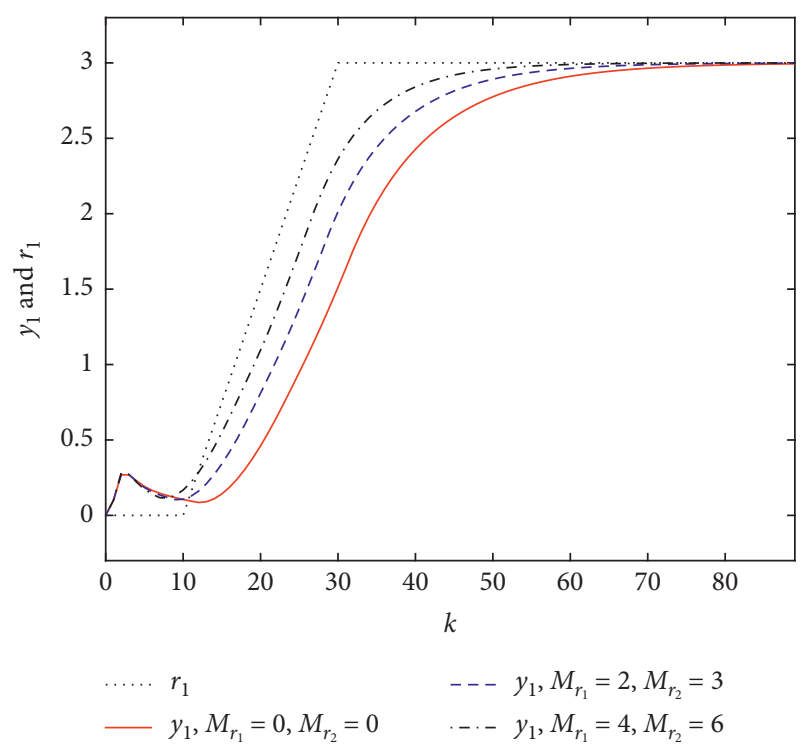

Figure 1: The output response of subsystem $S_{1}$.

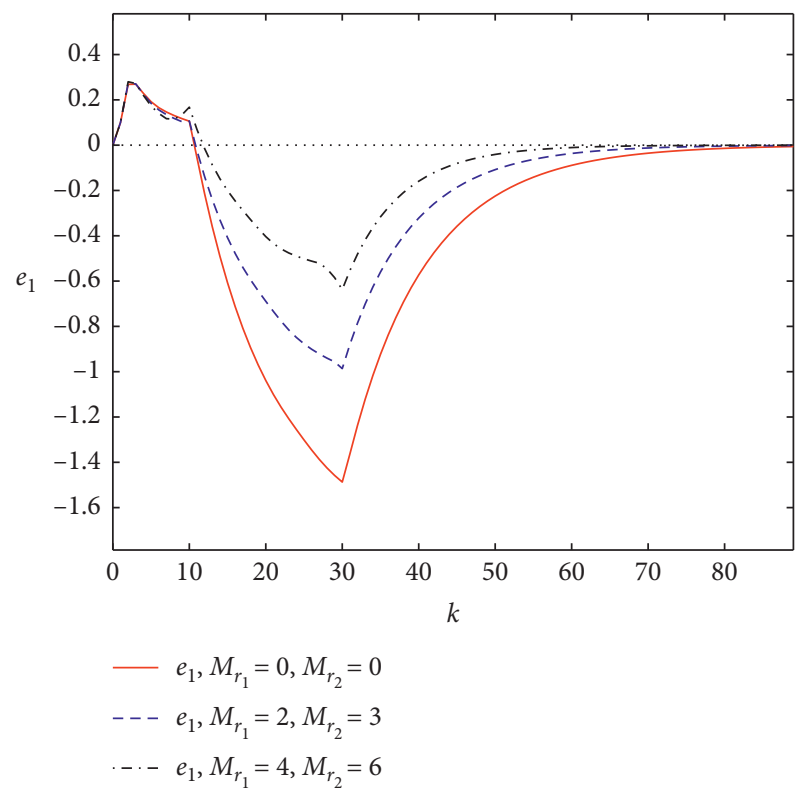

FIGURE 2: The tracking error response of subsystem $S_{1}$.

the effectiveness of the controller is evaluated and compared with classical tracking controller without preview action. It can be seen that all the performance indices values are reduced by using the preview controller. These merits mainly benefit from a novel preview compensation mechanism in the proposed tracking controller. The simulation results indicate that the suggested observer-based decentralized tracking controller with preview action has more remarkable and satisfactory control performance.

Example 2. To illustrate the application of the proposed control scheme, we consider the two identical pendulums

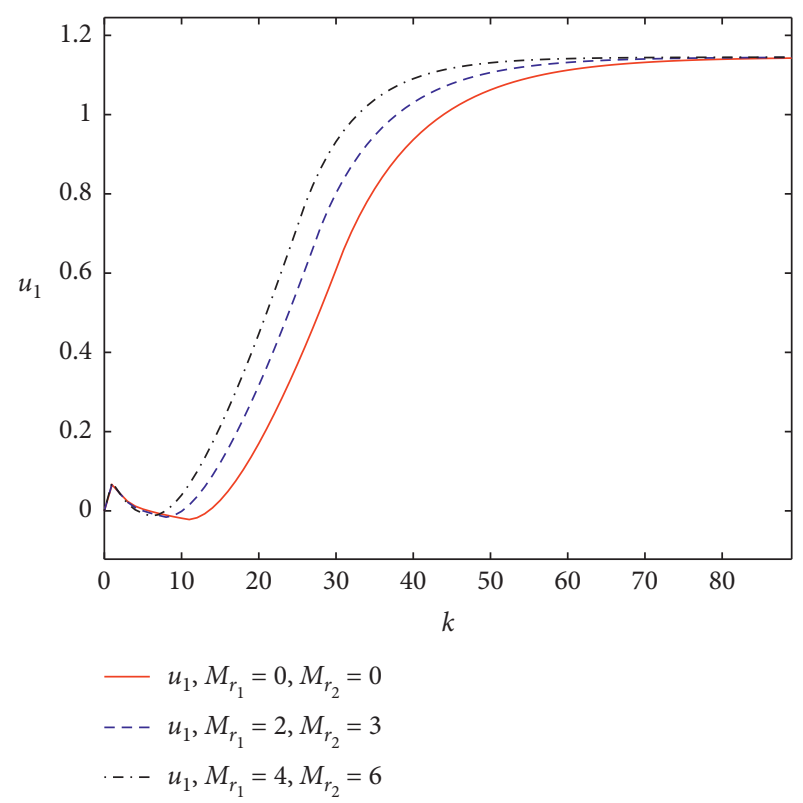

Figure 3: The control input response of subsystem $S_{1}$.

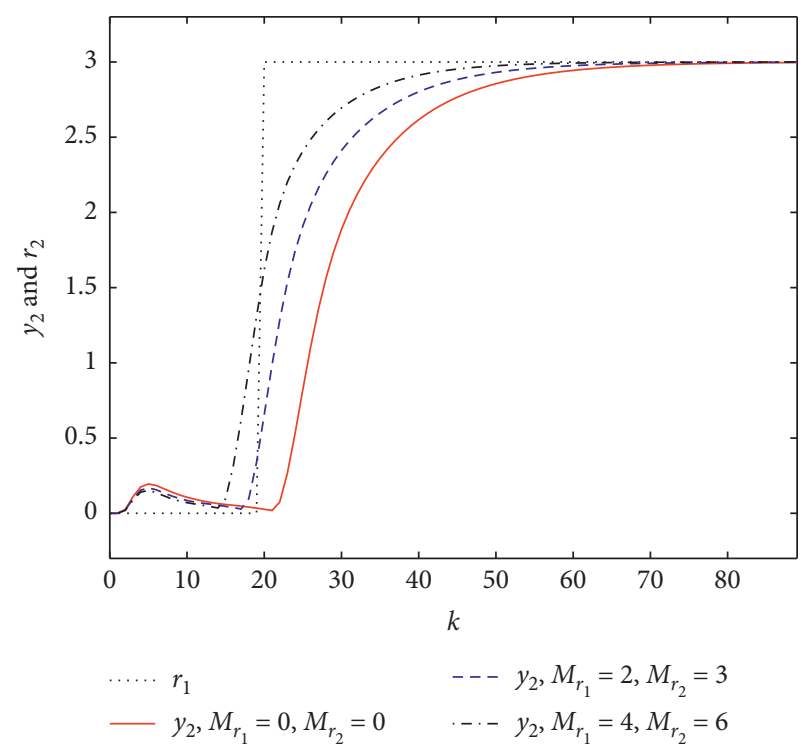

FIgURE 4: The output response of subsystem $S_{2}$.

which are coupled by a spring and subject to two distinct inputs [47] as shown in Figure 7.

The mathematical model of such an interconnected system can be described by

$$
\left\{\begin{array}{l}
\dot{x}_{1}(t)=A_{11} x_{1}(t)+B_{1} u_{1}(t)+A_{12} x_{2}(t) \\
y_{1}(t)=C_{1} x_{1}(t) \\
\dot{x}_{2}(t)=A_{22} x_{2}(t)+B_{2} u_{2}(t)+A_{21} x_{1}(t), \\
y_{2}(t)=C_{2} x_{2}(t)
\end{array}\right.
$$

with $x_{1}(t)=\left[\begin{array}{ll}\theta_{1}(t) & \dot{\theta}_{1}(t)\end{array}\right]^{T}, x_{2}(t)=\left[\begin{array}{ll}\theta_{2}(t) & \dot{\theta}_{2}(t)\end{array}\right]^{T}$, and 


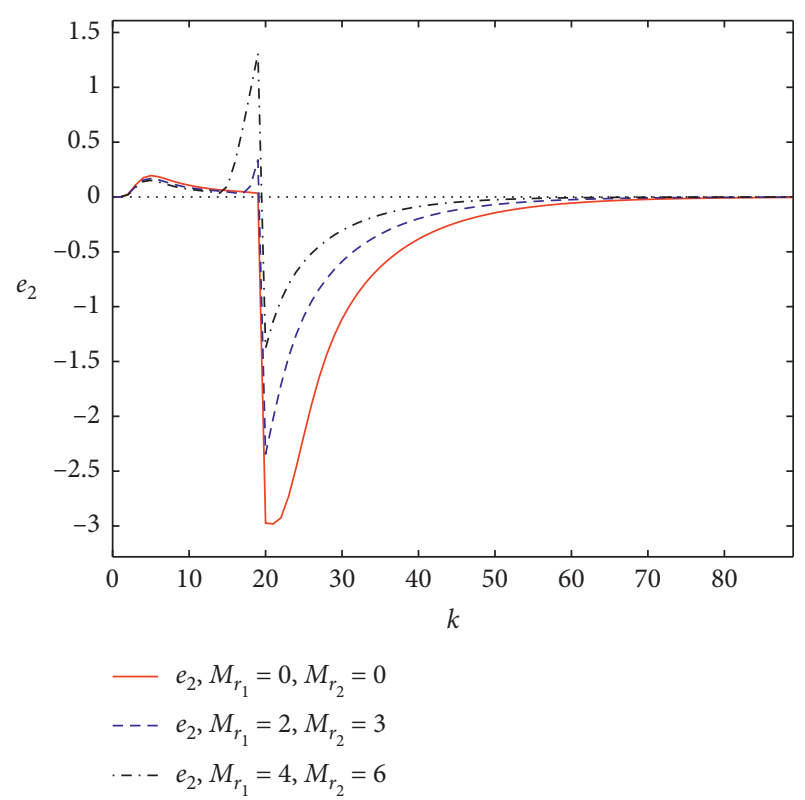

Figure 5: The tracking error response of subsystem $S_{2}$.

$$
\begin{aligned}
& A_{11}=A_{22}=\left[\begin{array}{cc}
0 & 1 \\
\frac{g}{l}-\frac{k a^{2}}{m l^{2}} & -\mu
\end{array}\right], \\
& B_{1}=B_{2}=\left[\begin{array}{c}
0 \\
\frac{1}{m l^{2}}
\end{array}\right], \\
& A_{12}=A_{21}=\left[\begin{array}{cc}
0 & 0 \\
\frac{k a^{2}}{m l^{2}} & 0
\end{array}\right], \\
& C_{1}=C_{2}=\left[\begin{array}{ll}
1 & 1
\end{array}\right],
\end{aligned}
$$

where $g$ represents the gravity, $\mu$ accounts for the friction, $m_{1}=m_{2}=m$ are the masses of both pendulums, and $k$ is the

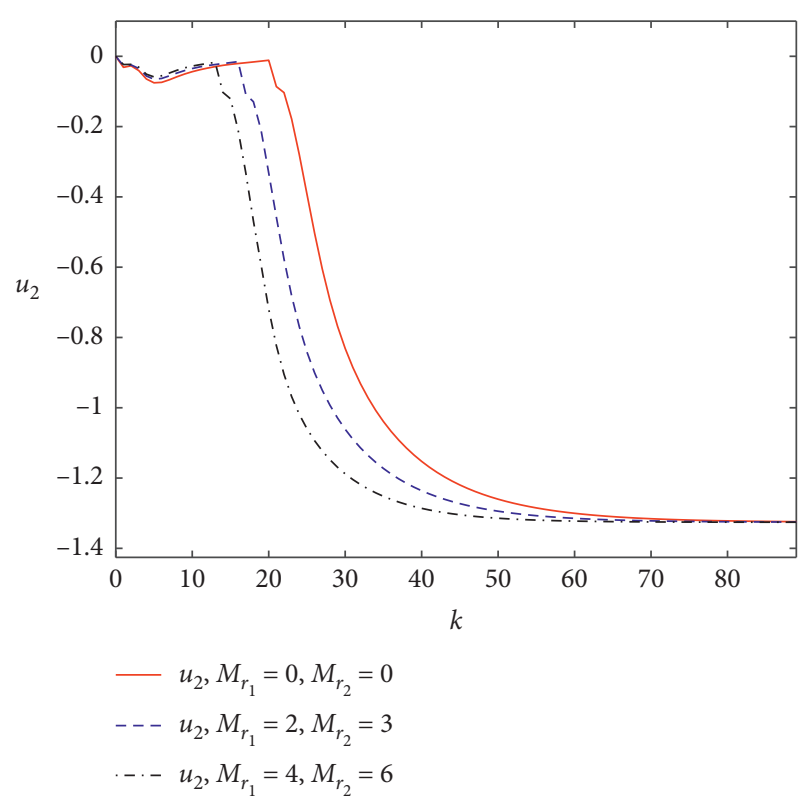

Figure 6: The control input response of subsystem $S_{2}$.

spring constant. The following parameters borrowed from [47] are used:

$$
\begin{aligned}
\frac{g}{l} & =1, \\
\frac{1}{m l^{2}} & =1, \\
\mu & =1, \\
\frac{k}{m} & =2, \\
\frac{a}{l} & =0.5 .
\end{aligned}
$$

When the sampling period is $0.25 \mathrm{~s}$, the approximate discrete-time model is of the following form:

$$
\begin{aligned}
& S_{1}:\left\{\begin{array}{l}
x_{1}(k+1)=\underbrace{\left[\begin{array}{cc}
1 & 0.25 \\
0.125 & 1
\end{array}\right]}_{A_{1}} x_{1}(k)+\underbrace{\left[\begin{array}{c}
0 \\
0.25
\end{array}\right]}_{B_{1}} u_{1}(k)+\underbrace{\left[\begin{array}{c}
0 \\
0.125 x_{21}(k)
\end{array}\right]}_{f_{1}\left(x_{1}(k), x_{2}(k)\right)} \\
y_{1}(k)=\underbrace{\left[\begin{array}{ll}
1 & 1
\end{array}\right] x_{1}(k) .}_{C_{1}}
\end{array}\right. \\
& S_{2}:\left\{\begin{array}{l}
x_{2}(k+1)=\underbrace{\left[\begin{array}{cc}
1 & 0.25 \\
0.125 & 1
\end{array}\right]}_{A_{2}} x_{2}(k)+\underbrace{\left[\begin{array}{c}
0 \\
0.25
\end{array}\right]}_{B_{2}} u_{2}(k)+\underbrace{\left[\begin{array}{c}
0 \\
0.125 x_{11}(k)
\end{array}\right]}_{f_{2}\left(x_{1}(k), x_{2}(k)\right)} \\
y_{2}(k)=\underbrace{\left[\begin{array}{ll}
1 & 1
\end{array}\right] x_{2}(k) .}_{C_{2}}
\end{array}\right.
\end{aligned}
$$


TABLe 1: Performance index results for subsystem $S_{1}$.

\begin{tabular}{|c|c|c|c|}
\hline Performance index & Classical controller $\left(M_{r_{1}}=0, M_{r_{2}}=0\right)$ & Preview controller $\left(M_{r_{1}}=2, M_{r_{2}}=3\right)$ & Preview controller $\left(M_{r_{1}}=4, M_{r_{2}}=6\right)$ \\
\hline IAE & 35.9558 & 22.8845 & 13.3241 \\
\hline ISE & 33.0938 & 14.0228 & 4.9148 \\
\hline ITAE & 1063.4 & 634.0 & 341.2 \\
\hline ITSE & 917.8741 & 374.7498 & 126.2100 \\
\hline
\end{tabular}

TABle 2: Performance index results for subsystem $S_{2}$.

\begin{tabular}{|c|c|c|c|}
\hline Performance index & Classical controller $\left(M_{r_{1}}=0, M_{r_{2}}=0\right)$ & Preview controller $\left(M_{r_{1}}=2, M_{r_{2}}=3\right)$ & Preview controller $\left(M_{r_{1}}=4, M_{r_{2}}=6\right)$ \\
\hline IAE & 35.5087 & 20.6670 & 14.2376 \\
\hline ISE & 61.1314 & 22.0606 & 9.9347 \\
\hline ITAE & 992.6933 & 548.3889 & 333.0283 \\
\hline ITSE & 1479.1 & 511.4 & 211.6 \\
\hline
\end{tabular}

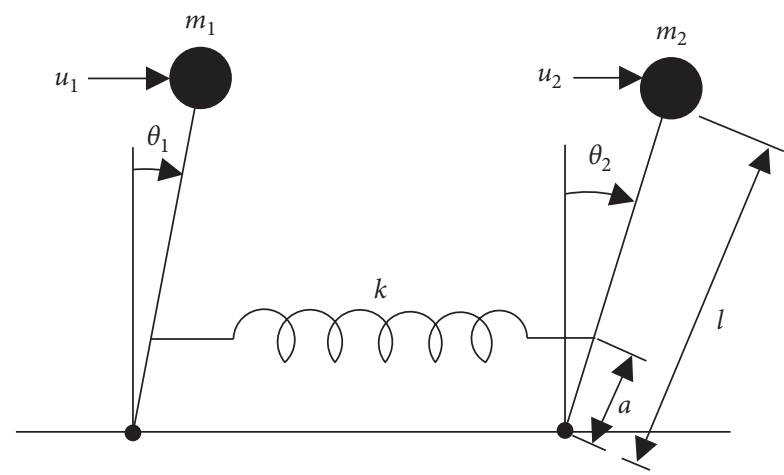

Figure 7: The coupled inverted pendulums.

In this example, the nonlinear interconnections $f_{i}\left(x_{1}, x_{2}\right)(i=1,2)$ degenerate into linear functions, and Assumption 1 holds with $\gamma_{1}=\gamma_{2}=0.125$. For simulation purpose, the desired trajectories $r_{1}(k)$ and $r_{2}(k)$ are taken as (63) and (64). Furthermore, their preview lengths are assumed to be $M_{r_{1}}$ and $M_{r_{2}}$, respectively.

To compare the effect of the preview action of the desired trajectory on the tracking performance, the following three cases are considered, including (i) $M_{r_{1}}=0, M_{r_{2}}=0$, (ii) $M_{r_{1}}=4, M_{r_{2}}=5$, and (iii) $M_{r_{1}}=5, M_{r_{2}}=9$. Given $\xi=1$, by resorting to the MATLAB LMI toolbox, the feasible solutions to LMI (30) in Theorem 1 can be derived. Furthermore, the observer gain and controller gain are designed as follows:

Case 1. When $M_{r_{1}}=0, M_{r_{2}}=0$, the decentralized observer gain and decentralized controller gain are

$$
\begin{aligned}
L_{1} & =\left[\begin{array}{l}
0.2881 \\
0.4844
\end{array}\right], \\
L_{2} & =\left[\begin{array}{l}
0.2881 \\
0.4844
\end{array}\right], \\
K_{e_{1}} & =-0.1014, \\
K_{e_{2}} & =-0.1014, \\
K_{\widehat{x}_{1}} & =[-8.4418-6.0674], \\
K_{\widehat{x}_{2}} & =[-8.4418-6.0674] .
\end{aligned}
$$

Case 2. When $M_{r_{1}}=4, M_{r_{2}}=5$, the decentralized observer gain and decentralized controller gain are

$$
\begin{aligned}
& L_{1}=\left[\begin{array}{l}
0.2895 \\
0.4861
\end{array}\right], \\
& L_{2}=\left[\begin{array}{l}
0.2838 \\
0.4772
\end{array}\right] \text {, } \\
& K_{e_{1}}=-0.1581 \text {, } \\
& K_{e_{2}}=-0.1611 \text {, } \\
& K_{\widehat{x}_{1}}=\left[\begin{array}{ll}
-8.5896 & -6.1464
\end{array}\right] \text {, } \\
& K_{\widehat{x}_{2}}=\left[\begin{array}{ll}
-8.6504 & -6.1626
\end{array}\right] \text {, } \\
& K_{r_{1}}=\left[\begin{array}{lllll}
0.1584 & 0.1577 & 0.1418 & 0.1326 & 0.1250
\end{array}\right] \text {, } \\
& K_{r_{2}}=\left[\begin{array}{llllll}
0.1614 & 0.1620 & 0.1470 & 0.1379 & 0.1304 & 0.1239
\end{array}\right] \text {. }
\end{aligned}
$$

Case 3. When $M_{r_{1}}=5, M_{r_{2}}=9$, the decentralized observer gain and decentralized controller gain are

$$
\begin{aligned}
& L_{1}=\left[\begin{array}{l}
0.2946 \\
0.4929
\end{array}\right], \\
& L_{2}=\left[\begin{array}{l}
0.2852 \\
0.4784
\end{array}\right] \text {, } \\
& K_{e_{1}}=-0.1987 \text {, } \\
& K_{e_{2}}=-0.2066 \text {, } \\
& K_{\widehat{x}_{1}}=\left[\begin{array}{ll}
-8.5990 & -6.1785
\end{array}\right] \text {, } \\
& K_{\widehat{x}_{2}}=\left[\begin{array}{ll}
-8.7559 & -6.2206
\end{array}\right] \text {, } \\
& K_{r_{1}}=\left[\begin{array}{llllll}
0.1990 & 0.1975 & 0.1743 & 0.1625 & 0.1534 & 0.1454
\end{array}\right] \text {, } \\
& K_{r_{2}}=\left[\begin{array}{llllll}
0.2065 & 0.2096 & 0.1887 & 0.1776 & 0.1692 & 0.1631
\end{array}\right. \\
& \left.\begin{array}{llllll}
0.1575 & 0.1521 & 0.1468 & 0.1408
\end{array}\right] \text {. }
\end{aligned}
$$

In numerical simulation, the initial states of the subsystems are $x_{1}=x_{2}=\left[\begin{array}{ll}0 & 0\end{array}\right]^{T}$, and the initial states of the observers are $\widehat{x}_{1}=\left[\begin{array}{ll}0.1 & 0\end{array}\right]^{T}$ and $\widehat{x}_{2}=\left[\begin{array}{ll}-0.1 & 0\end{array}\right]^{T}$. The simulation results are displayed in Figures $8-13$, from which 


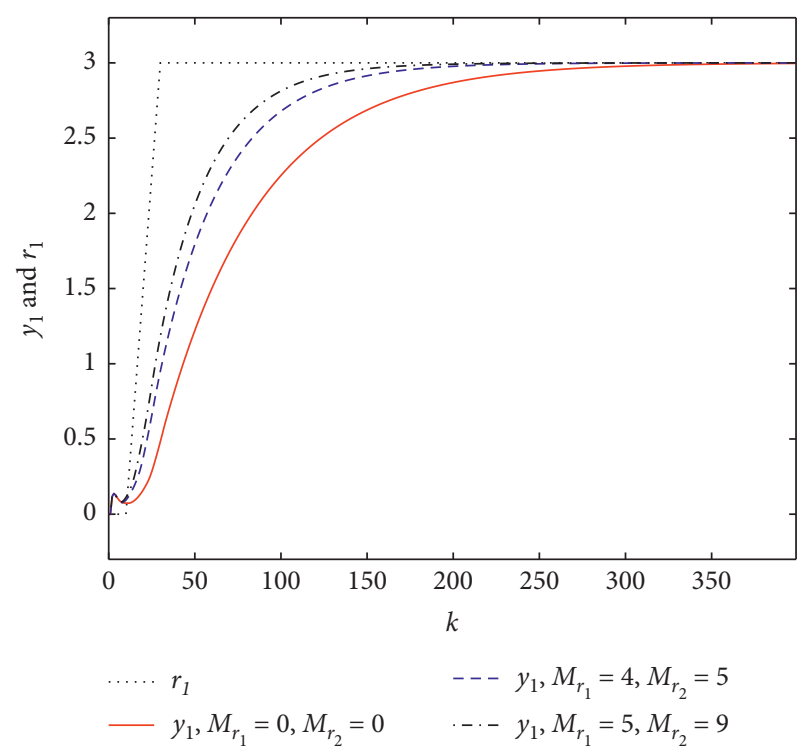

FIgURE 8: The output response of subsystem $S_{1}$.

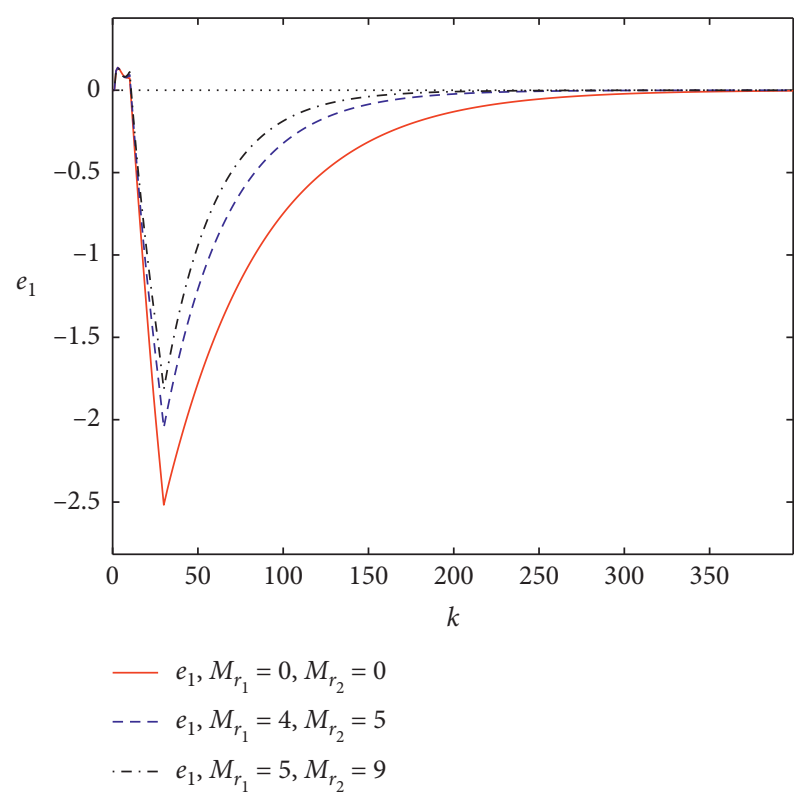

Figure 9: The tracking error response of subsystem $S_{1}$.

we can see that the proposed preview control scheme makes the closed-loop system produce faster response and higher tracking precision compared to the traditional controller without preview. Furthermore, Tables 3 and 4 give the comparison of the proposed scenario with the conventional method. The use of the preview information in tracking control structure largely improves the performance indices values. The simulation results confirm the effectiveness and superiority of our proposed preview control method.

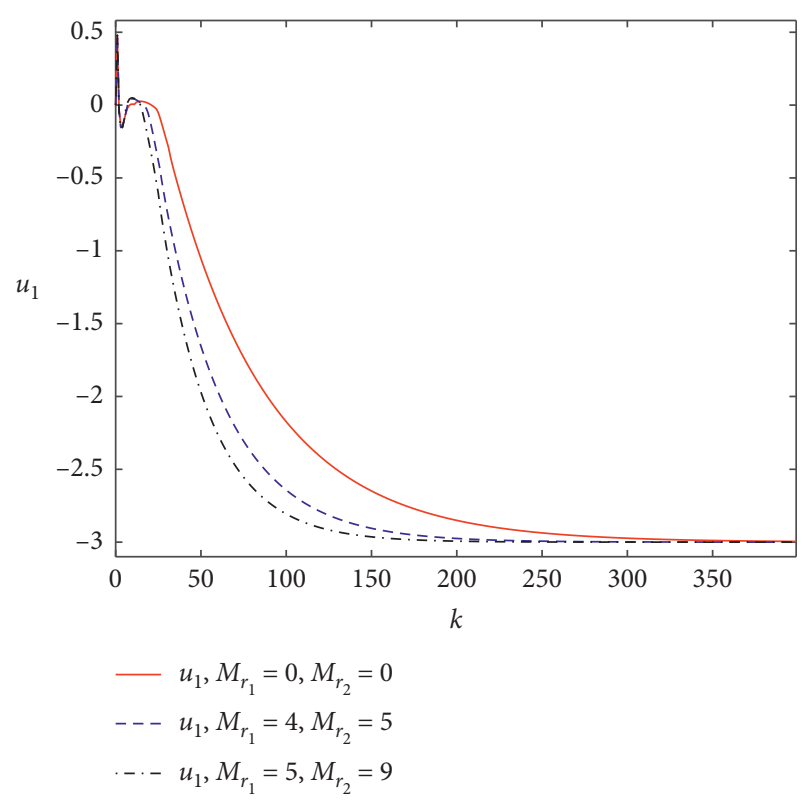

FIgURE 10: The control input response of subsystem $S_{1}$.

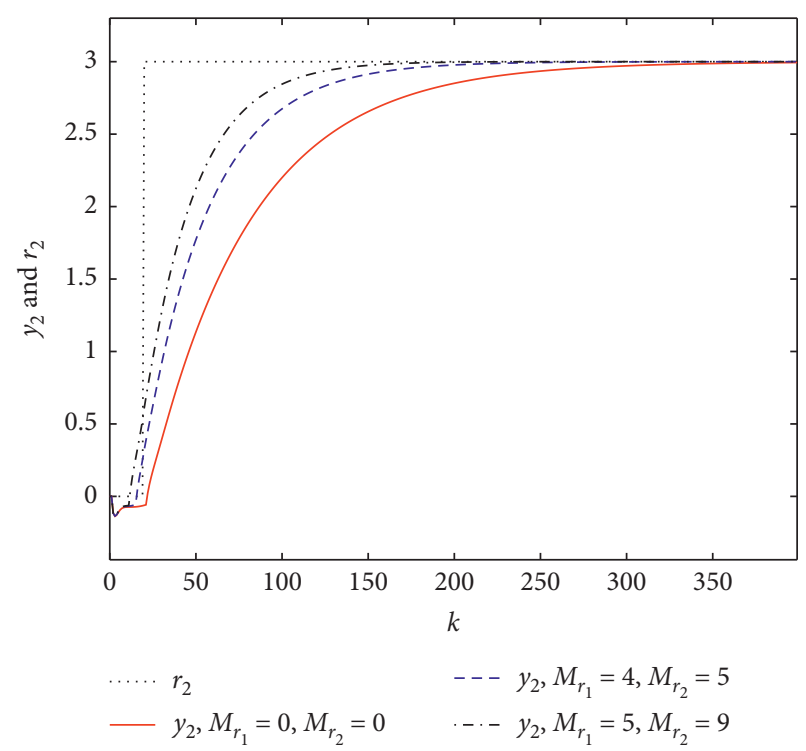

Figure 11: The output response of subsystem $S_{2}$.

\section{Conclusions}

In this paper, the problem of asymptotic tracking control with preview action for a class of interconnected nonlinear systems is investigated. By means of the difference approach, the augmentation technique, and the partition method, we construct a novel augmented error system where the known future knowledge of the desired trajectory is fully taken into account. Thereby, the output tracking problem is 


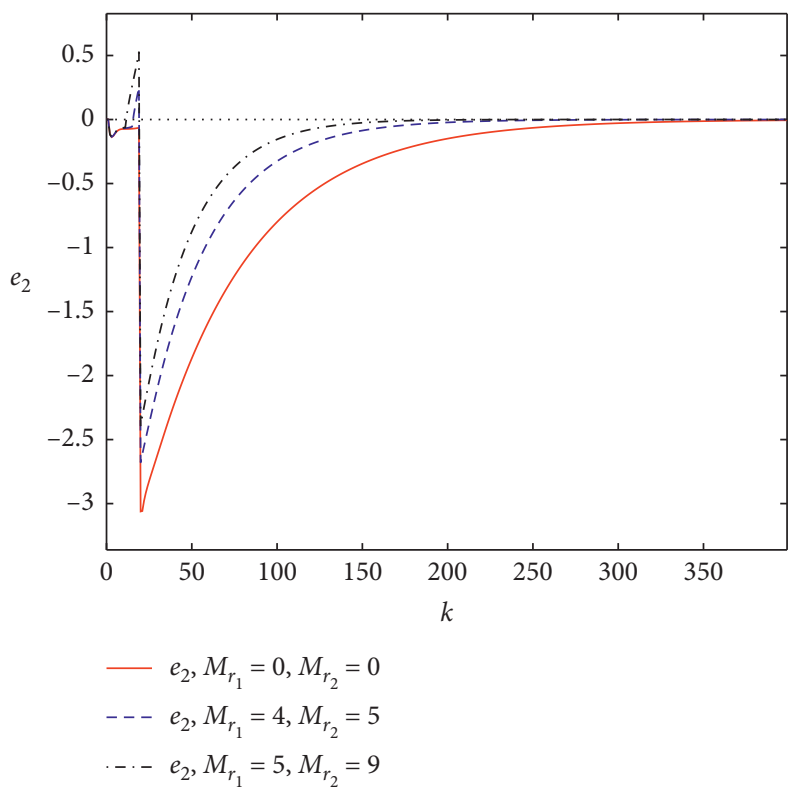

Figure 12: The tracking error response of subsystem $S_{2}$.

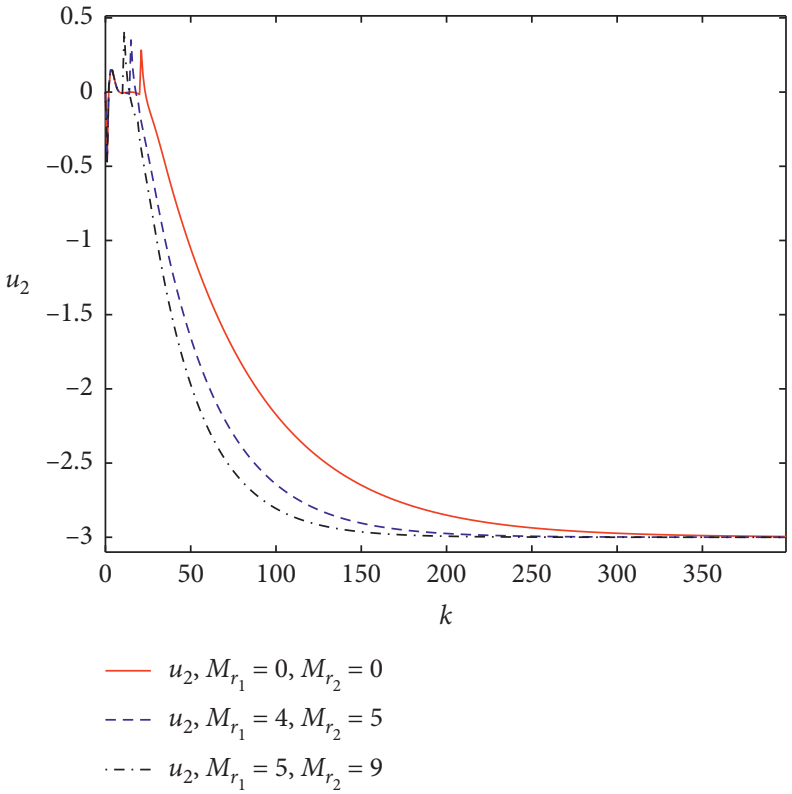

FIgURE 13: The control input response of subsystem $S_{2}$.

TABle 3: Performance index results for subsystem $S_{1}$.

\begin{tabular}{|c|c|c|c|}
\hline Performance index & Classical controller $\left(M_{r_{1}}=0, M_{r_{2}}=0\right)$ & Preview controller $\left(M_{r_{1}}=4, M_{r_{2}}=5\right)$ & Preview controller $\left(M_{r_{1}}=5, M_{r_{2}}=9\right)$ \\
\hline IAE & 171.5137 & 99.7774 & 75.6379 \\
\hline ISE & 227.8563 & 109.7278 & 73.8918 \\
\hline ITAE & 13137 & 5757 & 3876 \\
\hline ITSE & 11862 & 4631 & 2869 \\
\hline
\end{tabular}


Table 4: Performance index results for subsystem $S_{1}$.

\begin{tabular}{|c|c|c|c|}
\hline Performance index & Classical controller $\left(M_{r_{1}}=0, M_{r_{2}}=0\right)$ & Preview controller $\left(M_{r_{1}}=4, M_{r_{2}}=5\right)$ & Preview controller $\left(M_{r_{1}}=5, M_{r_{2}}=9\right)$ \\
\hline IAE & 186.1303 & 105.5119 & 75.2243 \\
\hline ISE & 287.7841 & 142.7642 & 90.1844 \\
\hline ITAE & 14490 & 5950 & 3515 \\
\hline ITSE & 14147 & 5482 & 3067 \\
\hline
\end{tabular}

transformed into a regulation problem. The proposed observer-based decentralized tracking controller design method is formulated by a feasibility problem involving an LMI, which can be efficiently computed through a one-step LMI procedure, to simultaneously determine the decentralized observation gain matrices and tracking controller gain matrices. Moreover, for each subsystem, the local tracking controller consists of the integral control action, the observer-based state feedback control action, and the preview action of the desired trajectory. Finally, the effectiveness of the suggested control approach is demonstrated by two numerical examples.

\section{Data Availability}

Data sharing is not applicable to this article as no datasets were generated or analysed during the current study.

\section{Conflicts of Interest}

The author declares that there are no conflicts of interest regarding the publication of this paper.

\section{Acknowledgments}

This research was supported by the Doctoral Research Fund in Shandong Jianzhu University and the National Natural Science Foundation of China (no. 61803228).

\section{References}

[1] X. Ma, H. Yu, Y. Wang, and Y. Wang, "Large-scale transportation network congestion evolution prediction using deep learning theory," PLoS One, vol. 10, no. 3, Article ID e0119044, pp. 1-17, 2015.

[2] Y. Wang, D. J. Hill, and G. Guo, "Robust decentralized control for multi-machine power systems," IEEE Transactions on Circuit and Systems, vol. 45, no. 3, pp. 271-279, 1998.

[3] S. Ke, W. Yu, T. Wang, and Y. Ge, "Aerodynamic performance and wind-induced effect of large-scale wind turbine system under yaw and wind-rain combination action," Renewable Energy, vol. 136, pp. 235-253, 2019.

[4] X. Zhang, M. Li, J. H. Lim et al., "Large-scale 3D printing by a team of mobile robots," Automation in Construction, vol. 95, pp. 98-106, 2018.

[5] Y. Yu, G. Lu, C. Sun, and H. Liu, "Robust backstepping decentralized tracking control for a 3-DOF helicopter," Nonlinear Dynamics, vol. 82, no. 1-2, pp. 947-960, 2015.

[6] G.-H. Yang and S.-Y. Zhang, "Decentralized robust control for interconnected systems with time-varying uncertainties," Automatica, vol. 32, no. 11, pp. 1603-1608, 1996.

[7] A. S. Tlili and N. Benhadj Braiek, " $H_{\infty}$ optimization-based decentralized control of linear interconnected systems with nonlinear interconnections," Journal of the Franklin Institute, vol. 351, no. 6, pp. 3286-3304, 2014.

[8] M.-L. Ni, J. Wang, and G. Li, "Decentralized tracking control for linear uncertain interconnected systems with time delays," Asian Journal of Control, vol. 10, no. 3, pp. 368-375, 2008.

[9] Q. Qu, H. Zhang, T. Feng, and H. Jiang, "Decentralized adaptive tracking control scheme for nonlinear large-scale interconnected systems via adaptive dynamic programming," Neurocomputing, vol. 225, pp. 1-10, 2017.

[10] H. Kheloufi, A. Zemouche, F. Bedouhene, C. Bennani, and H. Trinh, "New decentralized control design for interconnected nonlinear discrete-time systems with nonlinear interconnections," in Proceedings of the 2016 IEEE 55th Conference on Decision and Control, pp. 6030-6035, Las Vegas, NV, USA, December 2016.

[11] A. S. Tlili, "Linear matrix inequality robust tracking control conditions for nonlinear disturbed interconnected systems," Journal of Dynamic Systems, Measurement, and Control, vol. 139, no. 6, pp. 1-8, 2017.

[12] X.-J. Li, X.-X. Ren, and G.-H. Yang, "Backstepping-based decentralized tracking control for a class of interconnected stochastic nonlinear systems coupled via a directed graph," Information Sciences, vol. 477, pp. 302-320, 2019.

[13] Y.-F. Xie, W.-H. Gui, Z.-H. Jiang, and C. Huang, "Decentralized robust $H_{\infty}$ output feedback control for value bounded uncertain large-scale interconnected systems," International Journal of Control, Automation and Systems, vol. 8, no. 1, pp. 16-28, 2010.

[14] H. M. Soliman, H. A. Yousef, R. Al-Abri, and K. A. ElMetwally, "Decentralized robust saturated control of power systems using reachable sets," Complexity, vol. 2018, Article ID 2563834, 12 pages, 2018.

[15] H. Yang and S. Yin, "Descriptor observers design for Markov jump systems with simultaneous sensor and actuator faults," IEEE Transactions on Automatic Control, vol. 64, no. 8, pp. 3370-3377, 2019.

[16] H. Yang and S. Yin, "Reduced order sliding mode observerbased fault estimation for Markov jump systems," IEEE Transactions on Automatic Control, vol. 64, no. 11, pp. 4733-4740, 2019.

[17] H. Yang, Y. Jiang, and S. Yin, "Fault-tolerant control of timedelay Markov jump systems with Itô stochastic process and output disturbance based on sliding mode observer," IEEE Transactions on Industrial Informatics, vol. 14, no. 12, pp. 5299-5307, 2018.

[18] C. Dou, Z. Bo, X. Jia, D. Liu, and F. Zhao, "Fuzzy-observerbased robust tracking control for large interconnected power systems using WAMS," in Proceedings of the 2010 International Conference on Modelling, Identification and Control, pp. 460-465, Okayama, Japan, July 2010.

[19] O. El Maguiri, F. Giri, H. El Fadil, and F. Z. Chaoui, "Interconnected state observers for series resonant converters," IFAC Proceedings Volumes, vol. 42, no. 9, pp. 296301, 2009. 
[20] T. Tsuchiya and T. Egami, Digital Preview and Predictive Control (Translated by Fucheng Liao), Beijing Science and Technology, Beijing, China, 1994.

[21] N. Birla and A. Swarup, "Optimal preview control: a review," Optimal Control Applications and Methods, vol. 36, no. 2, pp. 241-268, 2015.

[22] Z. Zhen, "Research development in preview control theory and applications," Acta Automatica Sinica, vol. 42, no. 2, pp. 172-188, 2016.

[23] T. Katayama, T. Ohki, T. Inoue, and T. Kato, "Design of an optimal controller for a discrete-time system subject to previewable demand," International Journal of Control, vol. 41, no. 3, pp. 677-699, 1985.

[24] K. Takaba, "Robust servomechanism with preview action for polytopic uncertain systems," International Journal of Robust and Nonlinear Control, vol. 10, no. 2, pp. 101-111, 2000.

[25] J. Wu, F. Liao, and M. Tomizuka, "Optimal preview control for a linear continuous-time stochastic control systemin finite-time horizon," International Journal of Systems Science, vol. 48, no. 1, pp. 129-137, 2017.

[26] I. Youn, M. A. Khan, N. Uddin, E. Youn, and M. Tomizuka, "Road disturbance estimation for the optimal preview control of an active suspension systems based on tracked vehicle model," International Journal of Automotive Technology, vol. 18, no. 2, pp. 307-316, 2017.

[27] H. D. Choi, C. J. Lee, and M. T. Lim, "Fuzzy preview control for half-vehicle electro-hydraulic suspension system," International Journal of Control, Automation and Systems, vol. 16, no. 5, pp. 2489-2500, 2018.

[28] X. Zhang and X. Zhu, "Autonomous path tracking control of intelligent electric vehicles based on lane detection and optimal preview method," Expert Systems with Applications, vol. 121, pp. 38-48, 2019.

[29] X. Zhu, H. Zhang, J. Xi, J. Wang, and Z. Fang, "Robust speed synchronization control for clutchless automated manual transmission systems in electric vehicles," Proceedings of the Institution of Mechanical Engineers, Part D: Journal of Automobile Engineering, vol. 229, no. 4, pp. 424-436, 2015.

[30] L. M. Capisani, T. Facchinetti, and A. Ferrara, "Real-time networked control of an industrial robot manipulator via discrete-time second-order sliding modes," International Journal of Control, vol. 83, no. 8, pp. 1595-1611, 2010.

[31] S. Kajita, F. Kanehiro, K. Kaneko et al., "Biped walking pattern generation by using preview control of zero-moment point," in Proceedings of the 2003 IEEE International Conference on Robotics and Automation, pp. 1620-1626, Taipei, Taiwan, September 2003.

[32] A. Achnib, T.-B. Airimitoaie, P. Lanusse, S. Abrashov, M. Aoun, and M. Chetoui, "Discrete-time robust control with an anticipative action for preview systems," Journal of Dynamic Systems, Measurement, and Control, vol. 141, no. 3, pp. 1-11, 2019.

[33] W. Zhang, J. Bae, and M. Tomizuka, "Modified preview control for a wireless tracking control system with packet loss," IEEE/ASME Transactions on Mechatronics, vol. 20, no. 1, pp. 299-307, 2015.

[34] M. Yadegar, A. Afshar, and M. Davoodi, "Observer-based tracking controller design for a class of Lipschitz nonlinear systems," Journal of Vibration and Control, vol. 24, no. 11, pp. 2112-2119, 2018.

[35] C. E. de Souza and X. Li, "Delay-dependent robust $H_{\infty}$ control of uncertain linear state-delayed systems," Automatica, vol. 35, no. 7, pp. 1313-1321, 1999.
[36] X.-H. Chang, L. Zhang, and J. H. Park, "Robust static output feedback $H_{\infty}$ control for uncertain fuzzy systems," Fuzzy Sets and Systems, vol. 273, pp. 87-104, 2015.

[37] M. C. de Oliveira, J. C. Geromel, and J. Bernussou, "Extended $\mathrm{H}_{2}$ and $\mathrm{H}_{\infty}$ norm characterizations and controller parametrizations for discrete-time systems," International Journal of Control, vol. 75, no. 9, pp. 666-679, 2002.

[38] M. C. de Oliveira, J. Bernussou, and J. C. Geromel, "A new discrete-time robust stability condition," Systems \& Control Letters, vol. 37, no. 4, pp. 261-265, 1999.

[39] B. Baigzadehnoe, Z. Rahmani, A. Khosravi, and B. Rezaie, "Adaptive decentralized fuzzy output feedback tracking control for a class of nonlinear large-scale systems with input delays," Transactions of the Institute of Measurement and Control, vol. 40, no. 12, pp. 3534-3548, 2018.

[40] S. Dhbaibi, A. S. Tlili, S. Elloumi, and N. B Braiek, " $H_{\infty}$ decentralized observation and control of nonlinear interconnected systems," ISA Transactions, vol. 48, no. 4, pp. 458-467, 2009.

[41] A. S. Tlili, "Robustness design of a dynamic output-feedback decentralized controller using $H_{\infty}$ synthesis and LMI paradigm," International Journal of Control, Automation and Systems, vol. 15, no. 4, pp. 1544-1552, 2017.

[42] F. Angelini, C. D. Santina, M. Garabini, M. Bianchi, and G. M. Gasparri, "Decentralized trajectory tracking control for soft robots interacting with the environment," IEEE Transactions on Robotics, vol. 34, no. 4, pp. 924-935, 2018.

[43] R. Tang, X. Lü, Y. Zhai, and C. Gonge, "Optimal tracking control for nonlinear large-scale systems with persistent disturbances," Journal of Control Theory and Applications, vol. 8, no. 4, pp. 515-520, 2010.

[44] W. Gui, Y. Xie, N. Chen, and M. Wu, "The design of decentralized robust tracking controllers based on linear matrix inequality," Control Theory and Applications, vol. 17, no. 5, pp. 651-654, 2000.

[45] Y. X. Li and G. H. Yang, "Graph-theory-based decentralized adaptive output-feedback control for a class of nonlinear interconnected systems," IEEE Transactions on Cybernetics, vol. 49, no. 7, pp. 2444-2453, 2018.

[46] Y. Gao, X. Sun, and W. Wang, "Decentralized backstepping adaptive output tracking of large-scale stochastic nonlinear systems," Science China Information Sciences, vol. 60, no. 12, pp. 1-11, 2017.

[47] Q. Fu, P. Gu, and J. Wu, "Decentralized iterative learning control for large-scale interconnected linear systems with fixed initial shifts," International Journal of Control, Automation and Systems, vol. 15, no. 5, pp. 1991-2000, 2017. 